\title{
Non-radial Oscillations in Rotating Giant Planets with Solid Cores: Application to Saturn and its Rings
}

\author{
Jim Fuller ${ }^{\mathrm{a}, *}$, Dong Lai ${ }^{\mathrm{a}}$, Natalia I. Storch ${ }^{\mathrm{a}}$ \\ ${ }^{a}$ Center for Space Research, Department of Astronomy, Cornell University, Ithaca, NY 14853, USA
}

\begin{abstract}
Recent observations have revealed evidence for the global acoustic oscillations of Jupiter and Saturn. Such oscillations can potentially provide a new window into the interior structure of giant planets. Motivated by these observations, we study the non-radial oscillation modes of giant planets containing a solid core. Our calculations include the elastic response of the core and consider a wide range of possible values of the core shear modulus. While the elasticity of the core only slightly changes the frequencies of acoustic modes (including the f-modes), which reside mostly in the fluid envelope, it adds two new classes of shear modes that are largely confined to the core. We also calculate the effects of the Coriolis force on the planetary oscillation modes. In addition to changing the mode frequencies, the Coriolis force can cause the shear modes to mix with the f-modes. Such mixing occurs when the frequencies of the shear mode and the f-mode are close to each other, and results in "mixed modes" that have similarly large surface displacements and gravitational potential perturbations, but are slightly split in frequency. We discuss our results in light of the recent work by Hedman \& Nicholson (2013), which revealed the presence of density waves in Saturn's C-ring that appear to be excited by the gravitational perturbations associated with the f-mode oscillations within Saturn. We find that the fine splitting in wave frequencies observed in the rings can in principle be explained by the rotation-induced mixing between core shear modes and f-modes, possibly indicating the presence of a solid core within Saturn. However, in our current calculations, which assume rigid-body rotation and include only first-order rotational effects, significant fine-tuning in the planetary model parameters is needed in order to achieve these mode mixings and to explain the observed fine frequency splitting. We briefly discuss other effects that may modify the f-modes and facilitate mode mixing.
\end{abstract}

Keywords: Interiors; Jovian Planets; Resonances: rings; Saturn: interior; Saturn: rings

\section{Introduction}

Despite enormous advances in precision measurements of various global quantities of giant planets in our Solar System (mass, radius, rotation rate, gravitational moments, oblateness, etc.), our knowledge of the interior structure of these planets is rather limited. One uncertainty is the size of the central core, with estimates in the range of $\sim(0-10) M_{\oplus}$ (Guillot 2005) and $\sim(14-18) M_{\oplus}$ (Militzer et al. 2008) for Jupiter, and $\sim(9-22) M_{\oplus}$ (Guillot 2005) for Saturn. Another uncertainty concerns the mixing and possible stratification of heavy elements in the core and fluid envelope (Stevenson 1985; Leconte \& Chabrier 2012)

Global seismology is a promising technique for probing the internal structures of stars and planets. Indeed, the internal structure of the Earth, Moon, Sun, and numerous types of stars has been constrained primarily via measurements of global oscillations (see Unno et al. 1989 for stellar oscillations, Dahlen \& Tromp 1998, hereafter DT98, for a comprehensive description of the techniques of Earth seismology, and Chaplin \& Miglio 2013 a review of recent developments in asteroseismology). Unfortunately, direct detection of global oscillations in giant planets is extremely difficult because the oscillations produce negligible

\footnotetext{
* Corresponding author

Email address: derg@astro.cornell.edu (Jim Fuller)
} 
Table 1: Properties of the waves in Saturn's C-ring measured by HN13. The waves have the form $e^{i m \phi+i \sigma t}$, with the wave frequency $\sigma=|m| \Omega_{p}$. Resonant locations are measured from Saturn's center, and are taken from Baillie et al. (2011). The value of $|\delta \tau|$ is the approximate maximum semi-amplitude of the optical depth variation associated with each wave.

\begin{tabular}{ccccc}
\hline \hline Wave & Resonant location & $m$ & $\Omega_{p}(\mathrm{deg} /$ day $)$ & $|\delta \tau|$ \\
\hline W80.98 & $80988 \mathrm{~km}$ & -4 & 1660.3 & 0.09 \\
\hline $\mathrm{W} 82.00$ & $82010 \mathrm{~km}$ & -3 & 1736.6 & 0.07 \\
\hline $\mathrm{W} 82.06$ & $82061 \mathrm{~km}$ & -3 & 1735.0 & 0.21 \\
\hline $\mathrm{W} 82.21$ & $82209 \mathrm{~km}$ & -3 & 1730.3 & 0.15 \\
\hline $\mathrm{W} 84.64$ & $84644 \mathrm{~km}$ & -2 & 1860.8 & 0.09 \\
\hline W87.19 & $87189 \mathrm{~km}$ & -2 & 1779.5 & 0.14 \\
\hline \hline
\end{tabular}

luminosity perturbations and have small surface displacements (radial surface displacements are likely on the order centimeters). Recently, Gaulme et al. (2011) reported the detection of acoustic modes (p-modes) in the radial velocity data of Jupiter, but the quality of the data was insufficient to provide new constraints on Jupiter's interior model.

Saturn's ring system offers a unique opportunity to perform planetary seismology, because even mild gravitational perturbations associated with the planet's oscillation modes can generate density waves that propagate through the rings. Marley \& Porco (1993) investigated this idea in detail, arguing that some of the unexplained wave features (Rosen et al. 1991) in Saturn's C and D-rings were produced at Lindblad resonances with the gravitational perturbations associated with Saturn's oscillation modes. However, the existing Voyager data was insufficient to measure the properties of the waves, and so their seismic utility was limited.

Recently, Hedman \& Nicholson (2013) (hereafter HN13) used Cassini occultation data (see Colwell et al. 2009; Baillie et al. 2011) to measure the radial location of Lindblad resonance $\left(r_{L}\right)$, azimuthal pattern number $(m)$, and angular pattern frequency $\left(\Omega_{p}\right)$ of several waves in Saturn's C-ring. We retabulate the results of HN13 in Table 1. HN13 demonstrated that these waves can not be excited by resonances with any of Saturn's (known or unknown) satellites, but are compatible with being excited by low degree prograde sectoral $(l=|m|=2,3,4)$ fundamental oscillation modes (f-modes) of Saturn (as predicted by Marley 1991 and Marley \& Porco 1993). Throughout this paper, we adopt the convention that perturbations have the form $e^{i m \phi+i \sigma t}$, with the inertial frame mode frequency $\sigma=|m| \Omega_{p}>0$.

Intriguingly, HN13 found what appeared to be a "fine splitting" in the mode frequencies: Instead of one $m=-2$ wave excited by Saturn's $l=2, m=-2$ (prograde) f-mode, there are two discrete waves with a frequency difference of about $4 \%$; instead of one $m=-3$ wave excited by the $l=3, m=-3 \mathrm{f}$-mode, there are three waves with a frequency difference of $(0.1-0.3) \%$. No fine splitting was observed for the $m=-4$ wave, and no waves with $m<-4$ were observed.

The origin of these fine splittings is puzzling. Saturn rotates rapidly (with a spin period of 10.6 hours), which splits the $\mathrm{f}$-mode of a given degree $l$ into multiples with azimuthal order $m=-l,-l+1, \ldots, l-1, l$. For a given $m$, there are many f-modes that correspond to different degrees $l$ in the norotating configuration, but these modes differ in frequency by order unity (for small $l$ ), much larger than the observed splitting. Rotation can also introduce other Coriolis-force-supported modes (inertial modes and Rossby modes), but these modes all have frequencies (in the rotating frame) less than $2 \Omega_{s} \simeq 1630 \mathrm{deg} /$ day (where $\Omega_{s}$ is Saturn's rotation rate), which are smaller than the f-mode frequencies. We discuss some other possible effects in Section 6

In this paper, we explore the properties of oscillation modes of giant planets that contain a solid core. We have two goals. (1) Previous calculations of the oscillation modes of giant planets have been restricted to pure fluid models, with or without a dense core (e.g., Vorontsov \& Zharkov 1981, Vorontsov 1981, Marley 1991, Wu 2005, Le Bihan \& Burrows 2012). Although the global oscillations of the solid Earth are well studied (DT98) and there have been some studies on the effects of elasticity of the solid cores/crusts in white dwarfs and neutron stars (e.g., Hansen \& Van Horn 1979, McDermott et al. 1988, Montgomery \& 
Winget 1999), to our knowledge, no previous works have investigated the elastic response of a solid core in giant planets. The elasticity of a solid core adds entire new classes of modes that have previously been ignored and can also modify the properties of fluid modes (such as f-modes), which may have observable signatures. (2) We examine the possibility of rotational mixing between elastic core modes and envelope fmodes. While the influences of rotation on the mode frequencies are well studied in stars (Unno et al. 1989) and have been included in previous works on giant planetary oscillations (e.g., Vorontsov 1981; Marley 1991), the possibility of rotation-induced mode mixing has not been investigated. We show that such mode mixing can in principle lead to the appearance of multiple oscillation modes having very similar frequencies and characteristics. Nevertheless, as we show in this paper, significant fine tuning of the planetary model parameters is needed to produce the observed fine splitting of the waves in Saturn's rings.

Our paper is organized as follows. In Section 2, we generate simple giant planet models that will serve as the basis of our oscillation mode calculations. Section 3 describes the characteristics of oscillations in non-rotating planets, while Section 4 investigates mode mixing in rotating planets. In Section 5 , we calculate the effects of oscillation modes on Saturn's rings, and we compare our results to the observations of HN13. In Section 6, we summarize our results and discuss other effects (such as differential rotation and magnetic fields) that may modify the oscillation modes and influence mode mixing.

\section{Planetary Model}

Since our goal is to understand the effect of core elasticity on the oscillations of giant planets and to explore the possibility of rotation-induced mode mixing, we will not use sophisticated giant planet models with "realistic" equation of state (e.g., Guillot 2005) in this paper. Instead, we will consider simple planet models composed of a one-component solid core surrounded by a neutrally stratified fluid envelope charaterized by a $n=1(\Gamma=2)$ polytropic equation of state. These models allow us to capture the basic properties of giant planets without getting bogged down in uncertain details (e.g., helium rain out, liquid-metallic hydrogen phase transitions, core size and composition, etc.).

To generate our planet models, we first construct a polytropic model of index $n=1$ (so that the pressure is related to density as $P \propto \rho^{2}$ ). We then add a solid core by choosing a core radius, $R_{c}$, a dimensionless density enhancement $D$, and constant shear modulus $\mu$ (the shear modulus of the fluid envelope is zero). The density of material in the core is calculated by multiplying the density of material with $r<R_{c}$ in the original polytropic model by $D$. We then normalize the density profile so that the total mass $/ \mathrm{radius}$ equal the mass/radius of Saturn. With this density profile, we compute the gravitational acceleration via $g=G M(r) / r^{2}$, where $M(r)=\int_{0}^{r} 4 \pi r^{2} \rho d r$. We then assume the planet is neutrally stratified at all radii such that the Brunt-Vaisala frequency $N^{2}=0$. The pressure $P$ is obtained by integrating the hydrostatic equilibrium equation $d P / d r=-\rho g$, and the bulk modulus $K$ is given by

$$
K=-\rho g\left(\frac{d \ln \rho}{d r}\right)^{-1}
$$

The bulk modulus is related to the pressure $P$ via $K=\Gamma_{1} P$, with $\Gamma_{1}=d \ln P / d \ln \rho$.

For the purposes of calculating adiabatic acoustic-elastic pulsations in non-rotating spherically symmetric planets, a planetary model is completely described by three quantities as a function of radius: the density $\rho$, adiabatic bulk modulus $K$, and the shear modulus $\mu$ (see elastic oscillation equations in Section 3). Thus, our models have four free parameters: the density profile index ${ }^{1} n$, the radius of the solid core $R_{c}$ (or more precisely, the ratio of $R_{c}$ to the planet radius $R$ ), the core-envelope density jump $D$, and the (constant) core shear modulus $\mu$. These are ideally suited for understanding the basic characteristics of acousticelastic oscillations in giant planets. The $n=1$ polytropic density profile will generate realistic estimates of frequencies of p-modes propagating in the fluid envelope of the planet. The values of $R_{c}, D$, and $\mu$ affect the spectrum of modes propagating in the solid core, therefore, observations of these modes could provide strong constraints on core properties.

\footnotetext{
${ }^{1}$ Note that $n$ is the polytropic index (as in $P \propto \rho^{1+1 / n}$ ) of the planet without a core; when a core is added, the pressure and density in the fluid envelope no longer satisfies the polytropic relation.
} 


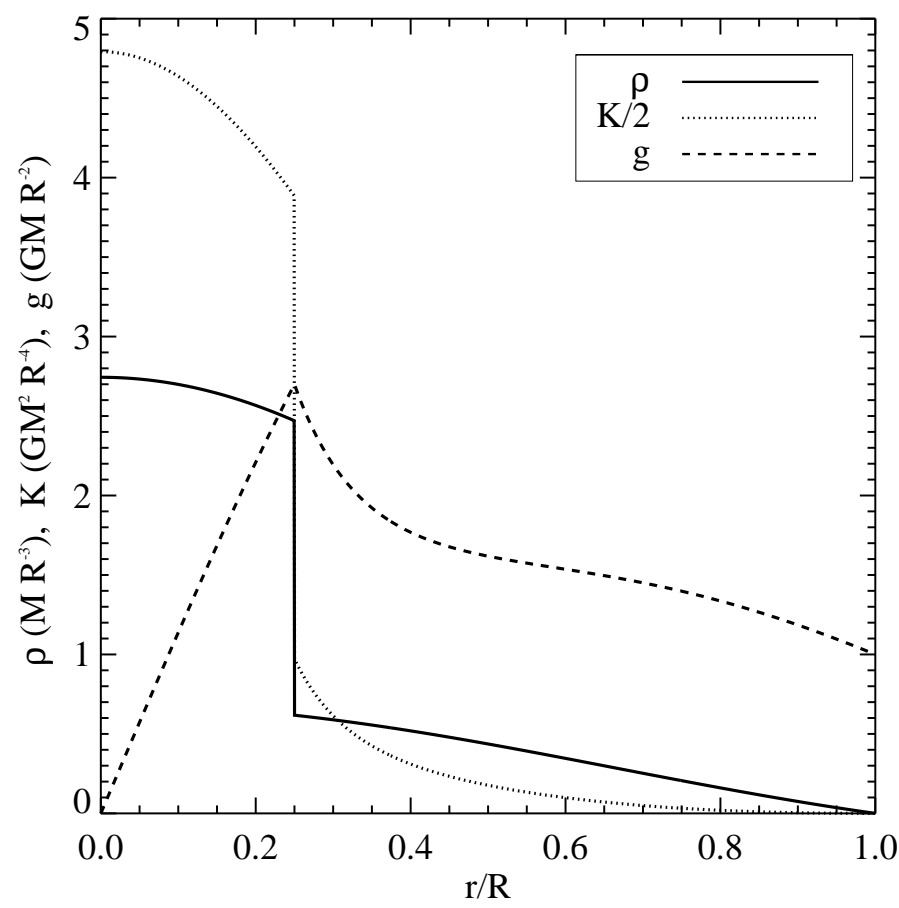

Figure 1: Density, bulk modulus and gravity profiles in our model of Saturn, with all quantities plotted in units with $G=M=R=1$. This model has an envelope density profile of a polytrope of index $n=1$, a core radius $R_{c}=0.25 R$ and a core density jump $D=4$. 
Figure 1 displays the structure of our primary planet model examined in this paper. It was constructed using $n=1, R_{c}=0.25 R$, and $D=4$. It has a core mass $M_{c}=16 M_{\oplus}$, central density $\rho_{c}=7.1 \mathrm{~g} / \mathrm{cm}^{3}$, and central pressure $P_{c}=2.2 \times 10^{12} \mathrm{~Pa}$. These values are consistent with the current observational constraints (see Guillot 2005).

\subsection{The Shear Modulus}

As will be shown below, the value of the shear modulus in the solid core determines the characteristics of elastic oscillation modes within Saturn. The core of Saturn is likely composed of ices and rocks. Assuming the core is at least partially solid, its shear modulus may be determined by the properties of high pressure ices (of which water ice is the dominant component).

Unfortunately, the shear modulus of water ice is unknown at the pressures $\left(P \sim 10^{12} \mathrm{~Pa}=10^{7}\right.$ bars $)$ and temperatures $\left(T \sim 8 \times 10^{3} \mathrm{~K}\right.$, Guillot 2005$)$ of Saturn's core. Asahara et al. (2010) found $\mu \approx 8 \times 10^{10} \mathrm{~Pa}$ for water ice at room temperature and pressures of $6 \times 10^{10} \mathrm{~Pa}$, well below the central pressure of Saturn. The measurements of Asahara et al. (2010) show the shear modulus of ice increasing toward larger pressures, suggesting $\mu>8 \times 10^{10} \mathrm{~Pa}$ in the core of Saturn. However, the shear modulus of many materials is also dependent on temperature. In the solid core of the Earth, the shear modulus of iron near its melting point is smaller than the shear modulus of iron at lower temperatures and pressures (Laio et al. 2000).

Recent molecular dynamics simulations (Cavazzoni 1999, French 2009, Militzer \& Wilson 2010, Militzer 2012, Hermann et al. 2011, Wang et al. 2011) have predicted multiple new phases of ice at $P \gtrsim 10^{11} \mathrm{~Pa}$. In particular, ice is likely in either a superionic or fluid phase at the core conditions of Saturn (French 2009, Wilson \& Militzer 2012), depending on the core temperature. In the superionic phase, the oxygen atoms form an ordered lattice, while the hydrogen atoms diffuse freely through the lattice. It is possible that the shear modulus of this superionic state is smaller than lower temperature phases of ice in which the oxygen-hydrogen bonds contribute to the strength of the lattice structure.

With such uncertainty in the shear modulus of material in a solid core of Saturn, we take the shear modulus to be a free parameter. In this work, we consider shear moduli in the range $2 \times 10^{8} \mathrm{~Pa}<\mu<$ $2 \times 10^{12} \mathrm{~Pa}$, or $10^{-4} \lesssim \mu / P \lesssim 1$, which seems to be a reasonable range given the above considerations. For simplicity, we also assume the value of the shear modulus is constant throughout the core.

\section{Oscillations of Non-rotating Planets}

The presence of a solid core affects planetary oscillations through the introduction of an elastic restoring force into the oscillation equations. The elastic force is characterized by the shear modulus $\mu$, whereas compressibility is characterized by the bulk modulus $K$. In a homogeneous elastic medium, there are two types of waves: (1) Sound waves (pressure waves or "p-waves"): these are longitudinal waves, with the displacement along the direction of wave vector $\mathbf{k}$, and the dispersion relation

$$
\omega^{2}=\frac{K+4 \mu / 3}{\rho} k^{2}
$$

(2) Shear waves ("s-waves"): these are transverse waves, with the displacement perpendicular to k, and the dispersion relation

$$
\omega^{2}=\frac{\mu}{\rho} k^{2}
$$

The two polarizations of s-waves are designated SH ("shear horizontal") and SV ("shear vertical") in Earth seismology. Obviously, shear waves do not exist in fluid regions.

In our planet models with a solid core, p-modes can propagate throughout the planet, with different sound speeds in the core and in the liquid envelope. The introduction of elastic restoring forces allows for two new types of modes to propagate in the solid core: spheroidal shear modes and toroidal shear modes, 
for whom the elasticity is the dominant restoring force. The p-modes and spheroidal shear modes (hereafter referred as "s-modes" ${ }^{2}$ are described by the displacement functions of the form

$$
\boldsymbol{\xi}(\mathbf{r})=U(r) Y_{l m}(\theta, \phi) \hat{\mathbf{r}}+V(r) r \nabla Y_{l m}(\theta, \phi) .
$$

The toroidal shear modes (hereafter refereed as "t-modes") are described by the toroidal displacement functions

$$
\boldsymbol{\xi}(\mathbf{r})=W(r) \boldsymbol{\nabla} \times\left[\mathbf{r} Y_{l m}(\theta, \phi)\right] .
$$

The t-modes exhibit no radial displacement, no gravitational perturbation, and are totally restricted to the solid regions of the planet. The s-modes, on the other hand, are slightly affected by the pressure perturbation, and can have a small, but finite gravitational perturbation. Each mode oscillates at its eigenfrequency $\omega$ such that

$$
\boldsymbol{\xi}(\mathbf{r}, t)=\boldsymbol{\xi}(\mathbf{r}) e^{i \omega t} \propto e^{i(m \phi+\omega t)} .
$$

With this convention, prograde modes with positive frequency have $m<0$, while retrograde modes with positive frequency have $m>0$.

Introducing the elastic forces into the oscillation equations makes them considerably more complicated. In Appendix A, we list the full adiabatic acoustic-elastic oscillation equations (see also Dahlen \& Tromp 1998, Alterman et al. 1959, Hansen \& Van Horn 1979, Montgomery \& Winget 1999) and boundary conditions. The spheroidal oscillations are described by a system of six linear coupled ordinary differential equations, while the toroidal oscillations are decoupled from the spheroidal oscillation equations and are described by two linear coupled ordinary differential equations. We choose to normalize our mode eigenfunctions via their inertia such that for any mode (indexed by $\alpha$ ),

$$
\int d V \rho \boldsymbol{\xi}_{\alpha} \cdot \boldsymbol{\xi}_{\alpha}^{*}=1
$$

with the integral extending over the volume of the planet.

Using relaxation techniques (see Press et al. 1998), we solve the acoustic-elastic oscillation equations for the spectrum of mode frequencies and eigenfunctions in a chosen planetary model. Figure 2 shows a plot of mode frequencies as a function of $l$, while Figure 3 shows the eigenfunctions of some oscillation modes of different types. Gas giant planetary models with a solid core and fluid envelope support p-modes that are largely restricted to the fluid envelope, and s-modes that are largely restricted to the solid core. The p-modes typically have large surface displacements and gravitational potential perturbations, while the s-modes have very small surface displacements and potential perturbations. The t-modes are very similar to the core s-modes, except that their displacement and potential perturbation are exactly zero in the fluid envelope. 3 by

Both the s-modes and t-modes satisfy the local dispersion relation (3). The radial wave number is given

$$
k_{r}^{2}=\frac{\rho}{\mu} \omega^{2}-\frac{l(l+1)}{r^{2}} .
$$

These modes propagate at the shear speed $v_{s}=\sqrt{\mu / \rho}$. A core with large density, small (but finite) shear modulus and large radius will support a dense spectrum of low frequency s-modes and t-modes. The frequency spacing of these modes is

$$
\Delta \omega \simeq \frac{\pi}{R_{c}} \sqrt{\frac{\mu}{\rho}}
$$

This frequency spacing is nearly independent of the mode index $\alpha$, and is dependent only upon the core radius, density, and shear modulus. In the asymptotic regime, s-modes and t-modes of the same $l$ are offset

\footnotetext{
${ }^{2}$ In the terminology of Earth seismology, the spheroidal shear modes correspond to SV modes and the toroidal shear modes correspond to $\mathrm{SH}$ modes.

${ }^{3}$ For a given $l$, the lowest-order s-mode corresponds to the Rayleigh wave that travels near the solid surface, while the lowest-order t-mode coresponds to the Love wave in the terminology of terrestrial seismology.
} 


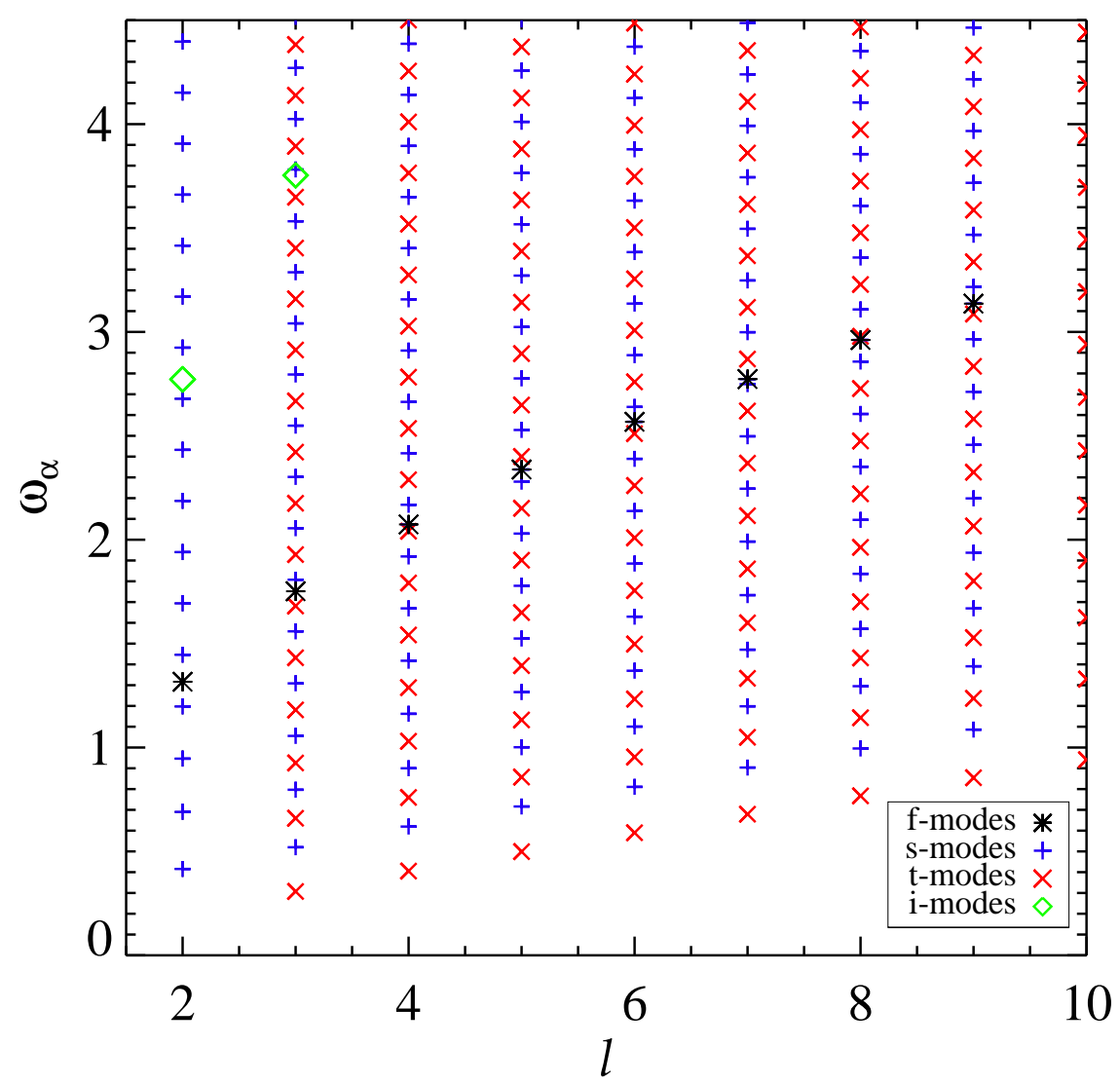

Figure 2: Oscillation mode spectrum of the planetary model with $n=1, R_{c}=0.25 R, D=4$, and $\mu=1.6$ GPa. The mode angular frequencies $\omega_{\alpha}$ (in units of $\sqrt{G M / R^{3}}$ ) are plotted as a function of the angular degree $l$. Only the fundamental p-modes (i.e. the f-modes) are shown, while higher-order p-modes are omitted for clarity. We have not included $l=2 \mathrm{t}$-modes because they cannot mix with even parity modes with $|m|>1$ (see Section 3.1. Note the nearly equal frequency spacing for both s-modes and t-modes at all values of $l$. 


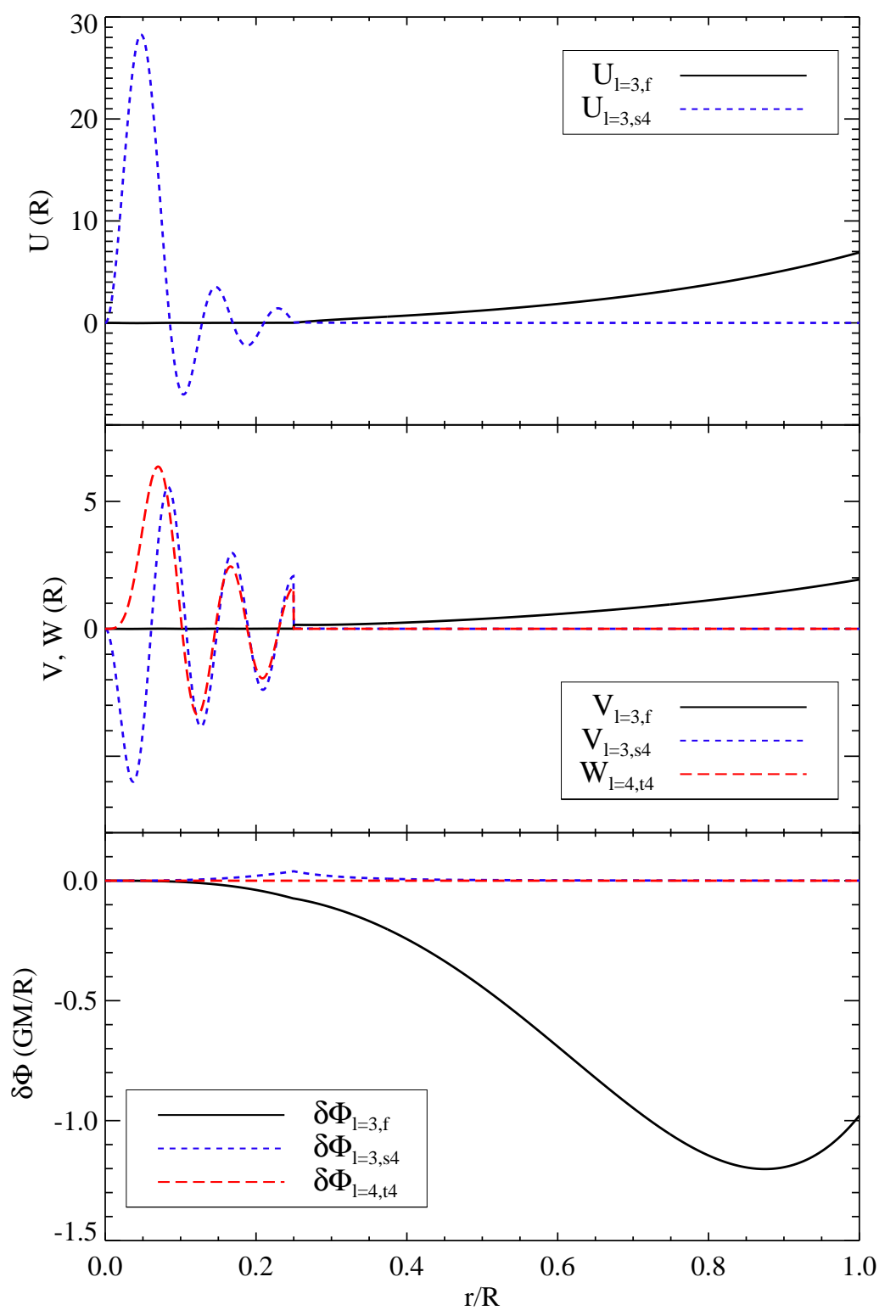

Figure 3: Mode eigenfunctions in the planetary model with $n=1, R_{c}=0.25 R, D=4$, and $\mu=1.6$ Gpa. The radial displacement $U_{\alpha}(r)$ (top panel), horizontal displacement $V_{\alpha}(r)$ or $W_{\alpha}(r)$ (middle panel), and gravitational potential perturbation $\delta \Phi_{\alpha}(r)$ (bottom panel) are shown. The different lines correspond to the $l=3 \mathrm{f}$-mode (black line, $\omega_{\alpha}=1.75$ ), a nearby $l=3$ spheroidal mode $\left(s 4\right.$, blue dashed line, $\left.\omega_{\alpha}=1.55\right)$, and an $l=4$ toroidal mode $\left(t 4\right.$, red dashed line, $\left.\omega_{\alpha}=1.54\right)$. 


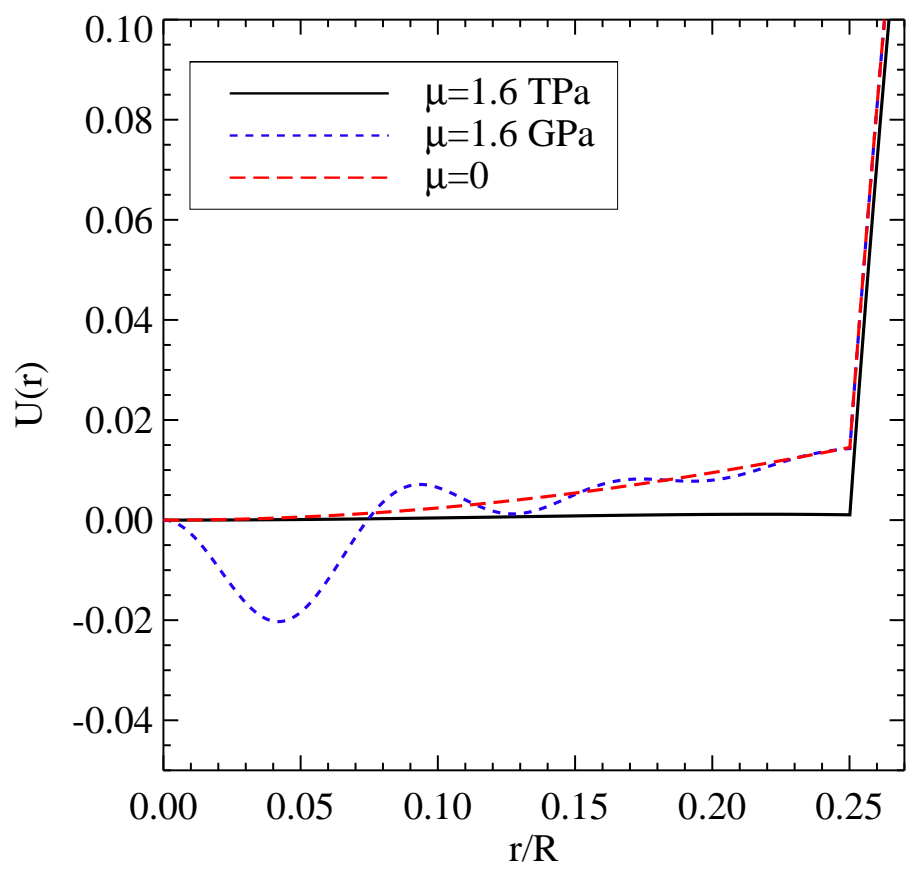

Figure 4: Radial displacements $U$ of the $l=3 \mathrm{f}$-mode for planetary models with differing values of the core shear modulus $\mu$. The other model parameters are the same as in Figure 3 The wave function for $\mu=1.6 \mathrm{GPa}$ obtains a wave-like character due to mixing with an s-mode (see Section 3.1). Note that the f-mode wave functions are nearly unaffected outside of the core. 


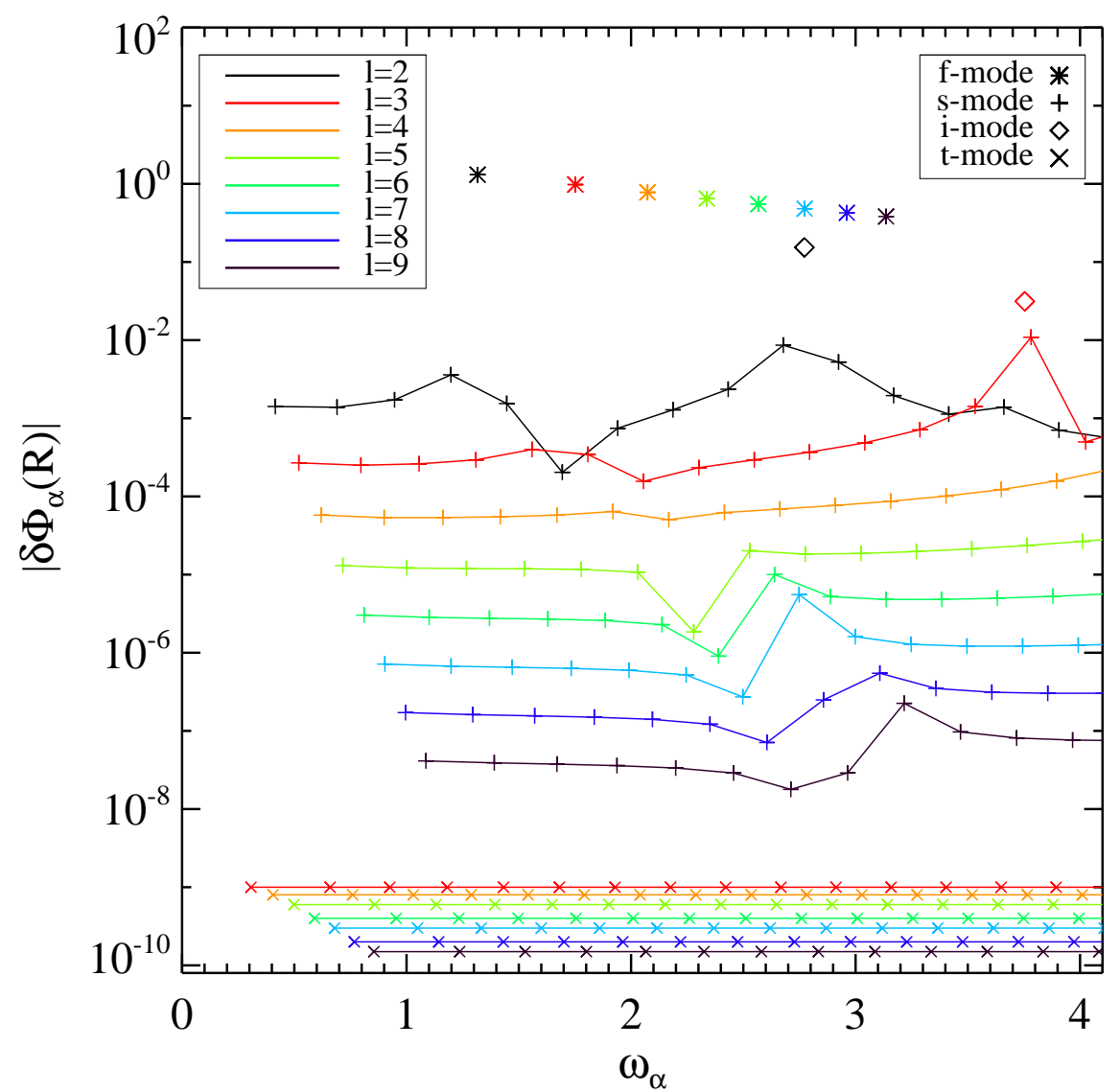

Figure 5: Surface gravitational potential perturbation, $\delta \Phi_{\alpha}(R)$, as a function of mode frequency $\omega_{\alpha}$ in the planetary model with $n=1, R_{c}=0.25 R, D=4$, and $\mu=1.6 \mathrm{GPa}$. S-modes and t-modes of equal value of $l$ have been connected by lines for clarity. The actual value of $\delta \Phi_{\alpha}(R)$ for the t-modes is exactly zero. The peaks in $\delta \Phi_{\alpha}(R)$ for s-modes are due to mixing with f-modes or i-modes. 
from one another by $\sim \Delta \omega / 2$ because of the differing boundary conditions at the core-envelope boundary ${ }^{4}$ Consequently, for s-modes of angular degree $l$, there exist t-modes of $l \pm 1$ with similar frequencies, and vice versa for t-modes.

The effect of a solid core on the properties of the f-mode and low order p-modes is miniscule. The main reason is that f-modes and low order p-modes have almost all of their inertia in the fluid envelope. Consequently, the value of the shear modulus has essentially no impact on the f-mode frequency (the the f-modes shown in Figure 4 differ by less than one part in $10^{5}$ in frequency), radial surface displacement, or potential perturbation 5 Nonetheless, the value of the shear modulus does affect the f-mode wave function in the core of the planet, as shown in Figure 4. For very rigid cores (large $\mu$ ), incoming waves are reflected, and the f-mode is excluded from the core. For softer cores (small but finite $\mu$ ), the f-mode may obtain a wave-like structure inside the core. Finally, for fluid cores, the f-mode penetrates into the core, but with a small amplitude, due to the jump in density.

In the absence of rotation, the external gravitational perturbation will be produced almost exclusively by envelope f-modes and low order p-modes (see Figure 5 6 6 The incompressive nature of low-order s-modes and t-modes creates zero (for t-modes) or very small density and gravity perturbations. Furthermore, because s-modes and t-modes are largely confined to the core, they produce very small fluid displacements at the surface. Thus, in the absence of rotation, s-modes and t-modes should be nearly impossible to detect.

Because our planetary models are neutrally stratified, they do not support g-modes. However, the discontinuity in density at the core-envelope boundary supports a single interface mode for each value of $l$. The frequency of this i-mode is given by

$$
\omega_{i}^{2} \approx \frac{\sqrt{l(l+1)} g_{c}}{R_{c}} \frac{\rho_{b}-\rho_{a}}{\rho_{b}+\rho_{a}}
$$

where $a$ and $b$ indicate that the quantity should be evaluated above and below the interface, respectively, and $g_{c}=g\left(R_{c}\right)$. For density jumps of $D \lesssim 2$ at the core-envelope boundary, the frequency of the interface mode may be comparable to that of the f-mode 7 The surface displacements and gravitational potential perturbations of the i-modes are typically greater than s-modes but less than f-modes for our planetary models.

\subsection{Mode Mixing}

The distinction between different types of modes (s-modes, t-modes, i-modes, and f-modes or p-modes) is not always clear. For a given planetary model, there may exist modes of nearly equal $\omega_{\alpha}$ and identical $l$ that will mix and obtain characteristics of two types of modes. This phenomenon is well known in Earth seismology (DT98), and is frequently observed as mixed modes in red giant stars (Chaplin \& Miglio 2013).

To understand the process of mode mixing in our non-rotating giant planet models (we examine rotationinduced mode mixing in Section 40, we calculate the modes for different values the shear modulus $\mu$. The frequencies $\omega_{\alpha}$ and surface displacements $U_{\alpha}(R)$ of some selected modes are shown in Figure 6. At certain shear moduli $\mu=\mu_{c}$, the f-mode and an s-mode have nearly identical frequencies. As the value of $\mu$ approaches $\mu_{c}$, the modes begin to exchange character with one another, causing the f-mode to penetrate into the core and the s-mode to penetrate into the fluid envelope. At $\mu=\mu_{c}$, the modes reach a minimum frequency separation and are equal superpositions of one another. As $\mu$ increases away from $\mu_{c}$, the mode

\footnotetext{
${ }^{4}$ For modes completely confined to the solid core, the boundary conditions at the core-envelope boundary imply $U(r) \simeq 0$ for the s-modes but $d W(r) / d r \simeq 0$ for the t-modes. Therefore, the number of wavelengths differs by about $1 / 4$ for s-modes and t-modes, accounting for the offset of $\sim \Delta \omega / 2$ between s-modes and t-modes in Figure 2

${ }^{5}$ An exception to this rule is at frequencies very near the avoided crossings with s-modes or i-modes, see Section 3.1

${ }^{6}$ Moreover, only relatively low-degree (low value of $l$ ) modes will produce significant external gravitational perturbations because the strength of the perturbation outside the planet falls off as $\delta \Phi(r)=\delta \Phi(R)(R / r)^{l+1}$, where $r$ is the distance from the center of the planet.

${ }^{7}$ Additional interface modes may also exist due to the presence of other density discontinuities in the planet. This may occur at the molecular-metallic hydrogen phase transition, the metallic hydrogen-molecular helium composition gradient, or at discontinuities in a differentiated core.
} 


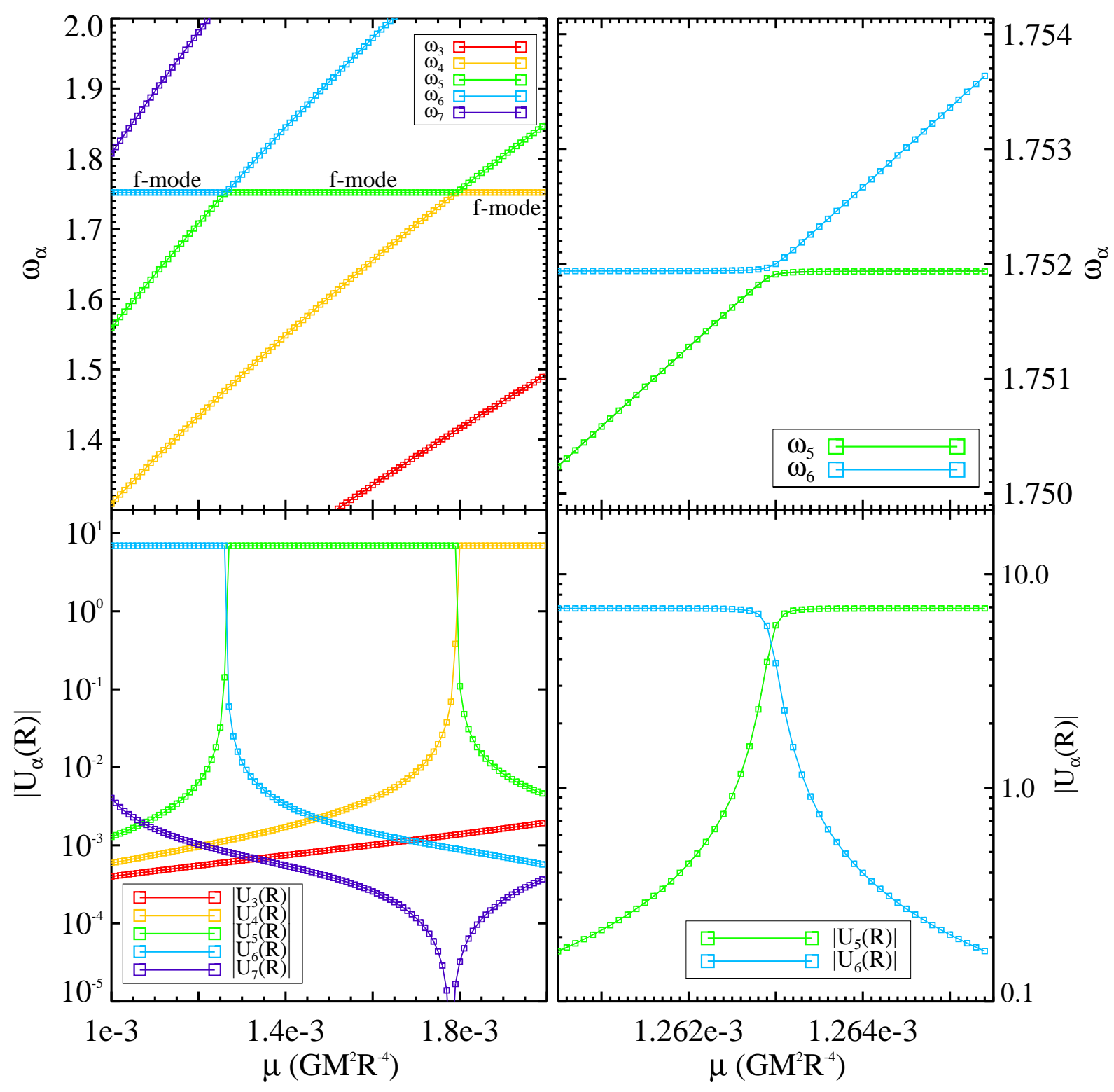

Figure 6: The frequencies $\omega_{\alpha}$ (top two panels) and radial surface displacements $U_{\alpha}(R)$ (bottom two panels) of a few selected modes for our planet models (as depicted in Figure 1), as a function of the value of the shear modulus $\mu$. Away from avoided crossings, the f-mode corresponds to the mode lying along the nearly horizontal line at $\omega_{\alpha} \simeq 1.75$, while the other modes are core s-modes. The right two panels present a zoom-n view of an avoided crossing between an s-mode and the f-mode. 
frequencies diverge from one another (note the frequencies are never exactly equal, resulting in an "avoided" crossing), having smoothly exchanged mode character. If a planetary model happens to contain modes near these avoided crossings, core s-modes may obtain substantially larger surface displacements and gravitational potential perturbations.

However, we find that it is unlikely for an s-mode and an f-mode to be near an avoided crossing in our planetary models, as evidenced by the extremely narrow width of the avoided crossings in Figure6. Avoided crossings between s-modes and i-modes have larger frequency widths, but will be difficult to observe due to the smaller surface displacements and potential perturbations of i-modes relative to f-modes. Finally, avoided crossings between i-modes and f-modes or p-modes have substantial frequency widths, but are unlikely to be observed because of the small number of i-modes (our models have only one i-mode for each value of $l$ ). We conclude that it is unlikely to see mode mixing phenomena (in the absence of rotation) in giant planets.

\section{Rotational Mode Mixing}

Thus far, we have considered the adiabatic acoustic-elastic oscillations of spherically symmetric, nonrotating planet models. However, the giant planets in our solar system spin rapidly, and the effects of rotation are quite important. For reference, Saturn's rotation period of $\simeq 11 \mathrm{hr}$ corresponds to $\Omega_{s} \simeq 0.36 \Omega_{\mathrm{dyn}}$ (where $\Omega_{\mathrm{dyn}}=\sqrt{G M / R^{3}}$ is the dynamical frequency of the planet), while the frequencies of the f-modes are typically $\omega_{f} \sim \sqrt{l} \Omega_{\mathrm{dyn}}$. Previous studies (e.g., Marley 1991 and Vorontsov \& Zharkov 1981) have shown that including rotational effects is essential in predicting the frequencies of f-modes of giant planets. Most studies of modes in rotating stars or planets utilize perturbation theory to obtain corrections to the frequencies and eigenfunctions of the modes, computed in powers of the small parameter $\lambda=\Omega_{s} / \omega_{0}$, where $\omega_{0}$ is the unperturbed mode frequency. To first order in $\lambda$, the only correction is from the Coriolis force, which splits the $2 l+1$ degenerate eigenfrequencies (corresponding to the $2 l+1$ values of $m$ for a given $l$ ) of the unperturbed planetary model. The second order rotational effects include the centrifugal force and the rotationally-induced planetary oblateness. In the absence of a solid core, the envelope p-modes are well separated in frequency, and non-degenerate perturbation methods suffice when calculating the influence of rotation.

However, in planetary models with a solid core, the addition of s-modes and t-modes may cause the spectrum of modes to become dense near the f-modes. In this case, the frequency spacing between modes may be smaller than the rotational corrections, and non-degenerate perturbation methods fail. Rotation not only shifts the frequencies of oscillation modes, but can also induce strong mode mixing. Spheroidal modes may acquire toroidal components, and vice versa. Both s-modes and t-modes may mix strongly with the f-mode, and a more precise treatment of the rotational effect is necessary. In this paper, we consider only the first order rotational effect (i.e., the Coriolis force), and assume uniform planetary rotation.

For two modes (labeled $\alpha$ and $\alpha^{\prime}$ ) to be mixed by the Coriolis force, the mixing matrix element (see Appendix B.2

$$
C_{\alpha \alpha^{\prime}}=i \int d V \rho \boldsymbol{\xi}_{\alpha}^{*} \cdot\left(\boldsymbol{\Omega}_{\boldsymbol{s}} \times \boldsymbol{\xi}_{\alpha^{\prime}}\right)
$$

where $\boldsymbol{\Omega}_{\boldsymbol{s}}$ is the spin vector, must be nonzero. This leads to some basic selection rules. First, only modes with $m=m^{\prime}$ will mix. Second, spheroidal modes only couple to other spheroidal modes with $l=l^{\prime}$, and likewise for toroidal modes. Finally, spheroidal and toroidal modes couple to one another only if $l=l^{\prime} \pm 1$. Thus, although a spheroidal mode of angular degree $l$ does not couple directly to other spheroidal modes of $l^{\prime}=l \pm 28$ they can couple indirectly through intermediary toroidal modes. We do not consider rotational mixing with inertial or Rossby modes (if they exist in Saturn's interior) because these modes have a maximum frequency of $\omega=2 \Omega_{s}$ in the rotating frame, and thus have smaller frequencies than any of Saturn's f-modes. Our method for calculating the effect of rotational mode mixing is outlined in Appendix B. Here we describe only the basic ideas.

\footnotetext{
${ }^{8}$ Second order rotational effects introduce direct coupling between modes with $l=l^{\prime} \pm 2$, but we consider only Coriolis coupling in this work.
} 
Consider a simple two mode system. In the absence of rotation, the two eigenmodes have frequencies $\omega_{1}$ and $\omega_{2}$, with eigenvectors $\mathbf{Z}_{1}$ and $\mathbf{Z}_{2}$. Including the Coriolis correction, we project the rotationally modified eigenmodes onto the original eigenmodes such that $\mathbf{Z}=a_{1} \mathbf{Z}_{1}+a_{2} \mathbf{Z}_{2}$ (see Appendix B). The eigensystem (equation B.16) describing the mixed modes is

$$
\left[\begin{array}{cc}
\bar{\omega}_{1}-\omega & C_{12} \\
C_{12}^{*} & \bar{\omega}_{2}-\omega
\end{array}\right]\left[\begin{array}{l}
b_{1} \\
b_{2}
\end{array}\right]=0
$$

where $b_{1}=\omega_{1} a_{1}, b_{2}=\omega_{2} a_{2}$, and $\bar{\omega}_{1}=\omega_{1}+C_{11}$ and $\bar{\omega}_{2}=\omega_{2}+C_{22}$ are the usual rotation-corrected mode frequencies when mixing is neglected. The Coriolis coupling coefficients $C_{\alpha \alpha^{\prime}}$ are defined in equation 11 . Defining $\Delta_{12}=\bar{\omega}_{1}-\bar{\omega}_{2}$, equation $(12)$ can be written as

$$
\left(\left[\begin{array}{cc}
\bar{\omega}_{1}+\bar{\omega}_{2}-2 \omega & 0 \\
0 & \bar{\omega}_{1}+\bar{\omega}_{2}-2 \omega
\end{array}\right]+\left[\begin{array}{cc}
\Delta_{12} & 2 C_{12} \\
2 C_{12}^{*} & -\Delta_{12}
\end{array}\right]\right)\left[\begin{array}{l}
b_{1} \\
b_{2}
\end{array}\right]=0 .
$$

This clearly shows that strong mode mixing occurs only if $2\left|C_{12}\right| \gtrsim\left|\Delta_{12}\right|$. The eigenfrequencies of equation (12) or 13 are

$$
\omega=\frac{\bar{\omega}_{1}+\bar{\omega}_{2}}{2} \pm \frac{1}{2} \sqrt{\Delta_{12}^{2}+4\left|C_{12}\right|^{2}}
$$

The corresponding eigenvectors are

$$
\mathbf{b}_{+}=\left[\begin{array}{c}
\cos \theta_{12} \\
x_{12}^{*} \sin \theta_{12}
\end{array}\right] \quad \text { and } \quad \mathbf{b}_{-}=\left[\begin{array}{c}
\sin \theta_{12} \\
-x_{12}^{*} \cos \theta_{12}
\end{array}\right],
$$

where $x_{12} \equiv C_{12} /\left|C_{12}\right| !^{9}$ and we have defined the mode mixing angle $\theta_{12}$ via

$$
\tan 2 \theta_{12}=\left|\frac{2 C_{12}}{\Delta_{12}}\right|
$$

When $2\left|C_{12}\right| \ll\left|\Delta_{12}\right|$ or $\theta_{12} \ll 1$, mode mixing is negligible, and the mode frequencies are simply $\bar{\omega}_{1}$ and $\bar{\omega}_{2}$. With decreasing $\left|\Delta_{12}\right|$ or increasing $\left|C_{12}\right|$, the mixing angle $\theta_{12}$ increases above zero, approaching $\pi / 4$ in the limit $2\left|C_{12}\right| \gg\left|\Delta_{12}\right|$. In this limit, the modified mode eigenfunctions are equal superpositions of the original mode eigenfunctions. Hence, if a core mode is strongly mixed to the f-mode, it will obtain the f-mode characteristics, including a much larger radial surface displacement $\left|\xi_{\alpha}(R)\right|$ and gravitational potential perturbation $\left|\delta \Phi_{\alpha}(R)\right|$. A "nearby" mode not strongly mixed or degenerate with the f-mode may still have an enhanced gravitational potential perturbation, with

$$
\delta \Phi_{\alpha} \simeq \frac{C_{\mathrm{f} \alpha}}{\Delta_{\mathrm{f} \alpha}} \delta \Phi_{\mathrm{f}}
$$

where $C_{\mathrm{f} \alpha}$ and $\Delta_{\mathrm{f} \alpha}$ are the mixing strength and frequency separation between the f-mode and the nearby mode. For planetary models in which the mode spectrum is dense near the f-mode, we should expect to see a peak in $\delta \Phi_{\alpha}$ centered on the f-mode, with a frequency width at its peak of order $2\left|C_{\mathrm{f} \alpha}\right|$.

\subsection{Strength of Coriolis Mode Mixing}

The importance of mode mixing due to the Coriolis force is determined by the value of $C_{\alpha \alpha^{\prime}}$. For most modes, the value of $\left|C_{\alpha \alpha^{\prime}}\right|$ is largest when $\alpha=\alpha^{\prime}$, i.e., due to self-coupling. This self-coupling term is identical to the standard rotationally induced frequency shift, i.e., if $\omega_{\alpha}$ is the frequency of mode $\alpha$ without rotation, then the Coriolis effect changes the frequency (in the rotating frame) to

$$
\bar{\omega}_{\alpha}=\omega_{\alpha}+C_{\alpha \alpha} .
$$

\footnotetext{
${ }^{9}$ The value of $C_{12}$ is real for spheroidal-spheroidal and toroidal-toroidal mode coupling (i.e., $x_{12}= \pm 1$ in this case), and is imaginary for spheroidal-toroidal mode coupling (i.e., $x_{12}= \pm i$ in this case).
} 
For s-modes and t-modes, the self-coupling coefficient has a value of $C_{\alpha \alpha} \approx m \Omega_{s} /[l(l+1)]$, while its value is typically of order $C_{\mathrm{ff}} \approx m \Omega_{s} / l$ for the f-modes in our planetary models. Thus, low-degree prograde sectoral $(m=-l)$ modes near the f-mode are typically reduced in frequency by a few $\times 10 \%$ in Saturn due to the effect of self-coupling.

In contrast to self-coupling, the mixing between core modes and envelope modes is typically quite weak in our planetary models. The main reason is simply that s-modes and t-modes have nearly all their inertia in the core, while f-modes and p-modes have nearly all their inertia in the envelope, and so the mode eigenfunctions do not have a large overlap in any region of the planet. We find typical values $\left|C_{\alpha \alpha^{\prime}}\right| \lesssim 10^{-3}|\mathrm{~m}| \Omega_{s}$ for mixing between core modes and f-modes of similar frequencies in our planetary models. These values decrease with decreasing shear modulus $\mu$ because of the increasingly oscillatory wave functions of the s-modes and tmodes. The value of $\left|C_{\alpha \alpha^{\prime}}\right|$ also decreases with increasing $l$ because f-modes of larger angular degree are confined closer to the planetary surface and have less inertia in the core. Therefore, the detuning $\Delta_{12}$ between a core mode and an f-mode must be very small for appreciable mixing to occur.

We also note that the mixing between s-modes and t-modes can be strong, with the values of $\left|C_{\alpha \alpha^{\prime}}\right|$ rising as large as the typical values for self-coupling. These large values of $\left|C_{\alpha \alpha^{\prime}}\right|$ occur for s-modes of angular degree $l$ and t-modes of angular degree $l \pm 1$ with the same number of nodes in their radial eigenfunctions. In non-rotating models, these modes typically have $\omega_{\alpha} \simeq \omega_{\alpha^{\prime}}$, however, their frequencies must be split by at least $\sim 2\left|C_{\alpha \alpha^{\prime}}\right|$ in rotating planets. Such strong mixing implies that each low-frequency core mode is a strong superposition of both spheroidal and toroidal components in a rapidly rotating planet.

\subsection{Two and Three-Mode Rotational Mixing}

Figure 7 shows an example of rotation-induced mixing between two modes. To make this figure, we consider the mixing between the $l=3, m=-3$ f-mode and the $l=4, m=-3$ t-mode depicted in Figure 3. At most spin frequencies, the modes are well-separated, and the mixing angle is small because of the small value of $C_{\mathrm{f}, \alpha}$. However, near a particular spin frequency the modes become nearly degenerate and mix strongly with one another. At maximum mixing, the modes consist of nearly equal superpositions of the original f-mode and t-mode, and there is the "avoided" crossing behavior of the frequencies. This is analogous to the mixing described in Section 3.1. Note that for a given f-mode and a nearby mode, strong mixing occurs over a very narrow range of spin frequency, with the width $\Delta \Omega_{s}$ of order $\left|C_{\mathrm{f} \alpha}\right|$, and $\left|C_{\text {f } \alpha}\right| / \Omega_{s} \ll 1$.

Three-mode mixing is more complex than two-mode mixing, and the general solution for a three mode eigensystem similar to equation 12 is sufficiently complicated that we do not attempt to analyze it here. Instead, we wish to understand the effect of rotational three-mode mixing between two core modes and an f-mode. For instance, there are many modes that do not couple directly to f-modes, but are coupled through an intermediary mode. One example is an $l=5, m=-3 \mathrm{~s}$-mode mixed with a $l=4, m=-3$ t-mode, which in turn is mixed with the $l=3, m=-3 \mathrm{f}$-mode. We detail a solution of such a three-mode system in Appendix C. The main result is that the $l=5 \mathrm{~s}$-mode can strongly mix with the f-mode even though they are not coupled directly. Thus, we expect that modes nearly degenerate with an f-mode will obtain substantial gravitational potential perturbations, even if they are not directly coupled with the f-mode.

We obtain the exact numerical solution to such a three-mode system and plot the results in Figure 8 . As expected, both core modes undergo avoided crossings with the f-mode in which they mix strongly with it. We also note that the strong mode mixing between the t-mode and s-mode causes their frequencies to diverge away from one another as the spin frequency is increased 10 This mode "repulsion" affects the location of avoided crossings with the f-mode, causing them to occur at a spin frequency different than one would expect from a two mode analysis. Finally, only the mode whose frequency is nearly degenerate with that of the f-mode is affected by the avoided crossing. Therefore, modes that differ substantially in frequency from an f-mode will not strongly mix with it.

\footnotetext{
${ }^{10}$ In a real system, these modes will also mix strongly with other s-modes and t-modes, which will mitigate this frequency divergence. Hence, it is important to extend mode mixing calculations to large values of $l$ in order to capture the realistic mixing behavior.
} 


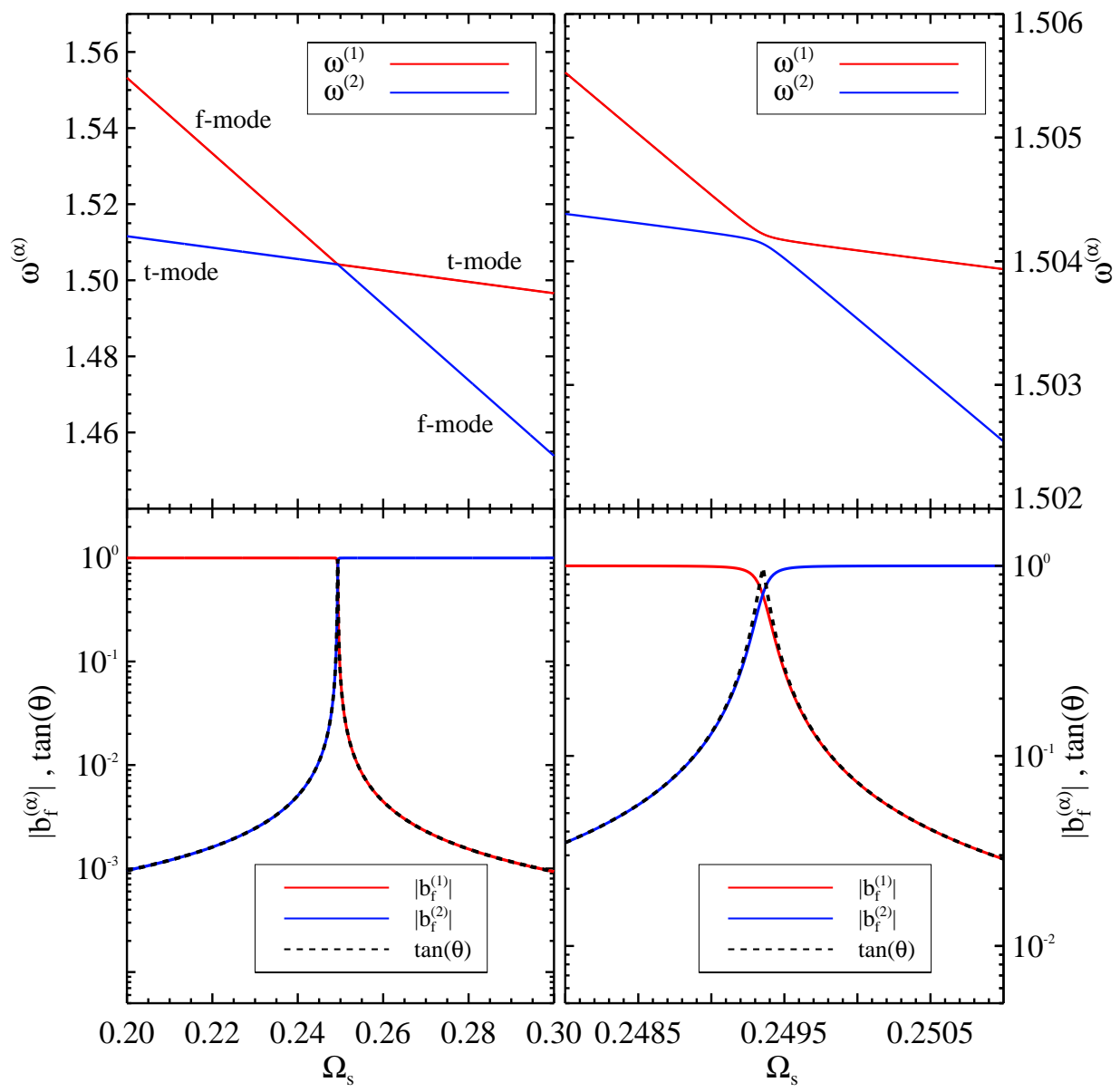

Figure 7: Top: The frequencies $\omega^{(\alpha)}$ of the rotationally mixed $l=3 \mathrm{f}$-mode and an $l=4 \mathrm{t}$-mode as a function of Saturn's spin frequency $\Omega_{s}$ (in units of $\sqrt{G M / R^{3}}$ ). Bottom: The projections $b_{\mathrm{f}}^{(\alpha)}$ of each rotationally mixed mode onto the f-mode. We have also plotted the mode mixing angle $\tan (\theta)$. The modes have an avoided crossing at $\Omega_{s} \simeq 0.25$, where they are maximally mixed. The "f"-mode corresponds to $\omega^{(1)}$ for $\Omega_{s} \lesssim 0.25$, and corresponds to $\omega^{(2)}$ for $\Omega_{s} \gtrsim 0.25$. The right panels show a zoom-in on the left panels. The eigenmode amplitudes have been normalized via $\sum_{\beta}\left|b_{\beta}^{(\alpha)}\right|^{2}=1$. 


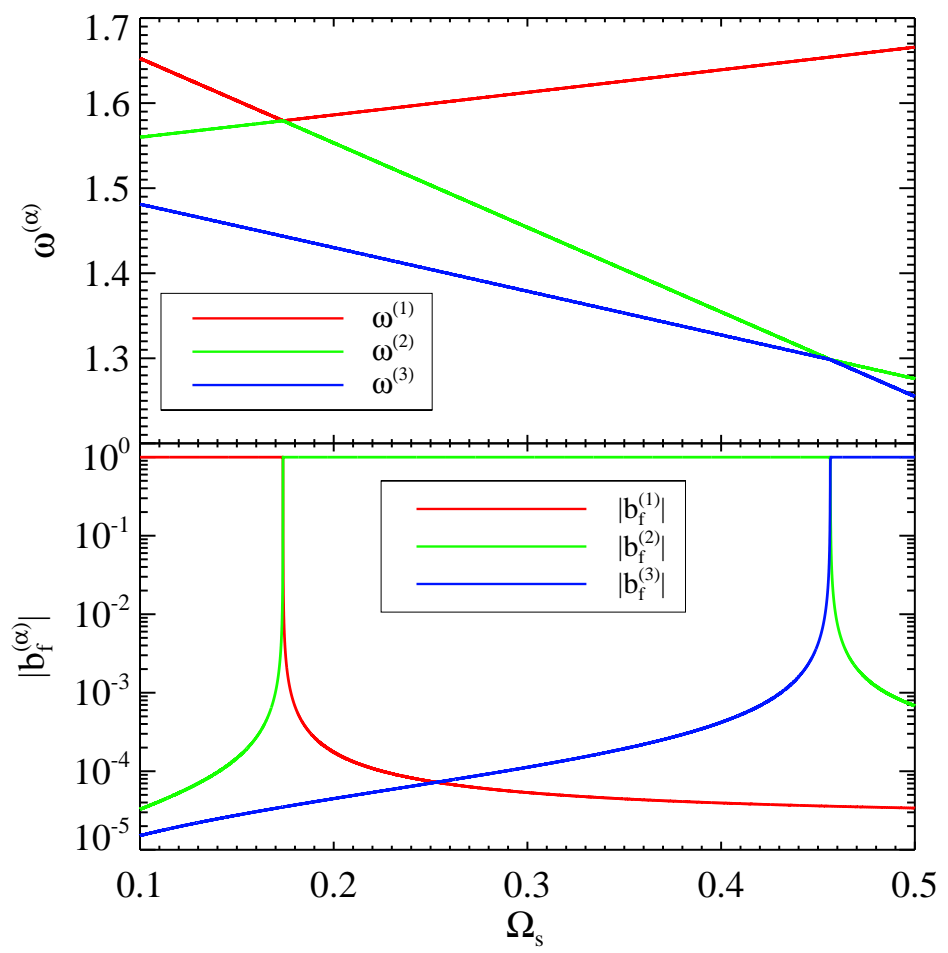

Figure 8: Same as Figure7 but for a system of three mixed modes. Away from the avoided crossings, the "f"-mode corresponds to the line with the steepest slope. The "s"-mode and "t"-mode are strongly mixed at all values of $\Omega_{s}$, but only mix significantly with the " $\mathrm{f}$ "-mode near the avoided crossings. 


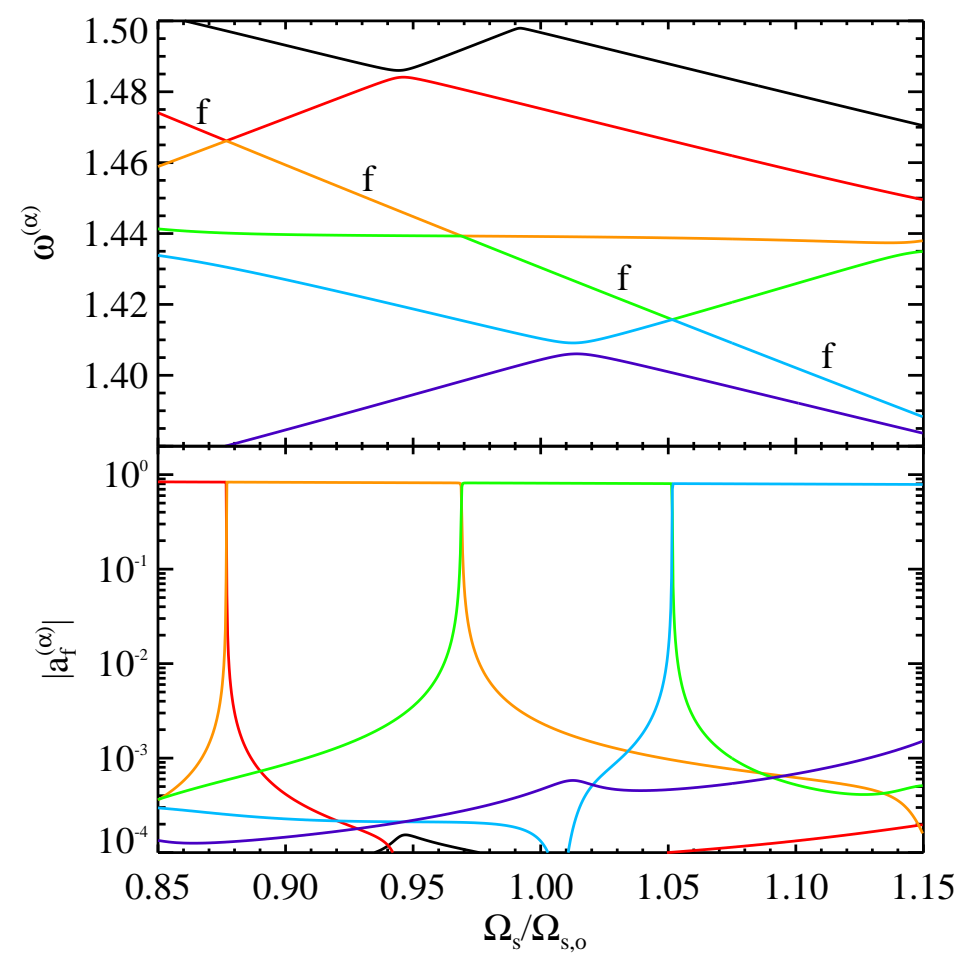

Figure 9: Same as Figure 8 but for a system containing 112 modes (only the six modes with frequency nearest the f-mode are shown). Here we plot the projection onto the f-mode, $a_{\mathrm{f}}^{(\alpha)}=b_{\mathrm{f}}^{(\alpha)} / \omega^{(\alpha)}$, with the modes normalized via equation B.22, The spin frequency $\Omega_{s}$ is plotted in units of Saturn's actual spin frequency. We have incorporated modes up to $l=18$, and have included all modes with frequency in the vicinity of the $l=3 \mathrm{f}$-mode. The "f"-mode is labeled away from the avoided crossings.

\subsection{Multi-Mode Rotational Mixing}

We can now try to understand the effects of mode mixing in more realistic systems, which may contain hundreds of coupled modes. To this end, we solve equation (B.16) for a system containing 112 modes for our planetary model with a fixed $\mu=1.6 \mathrm{GPa}$ but with a range of spin frequencies. We include all the modes with frequency comparable to the $l=3 \mathrm{f}$-mode; this requires the inclusion of modes up to $l=18$. The results are shown in Figure 9. The qualitative features of the mode mixing are similar to the two and three-mode mixing shown in Figures 7 and 8 . Although many modes are capable of mixing with the f-mode, the small coupling coefficients $C_{\mathrm{f} \alpha}$ ensure that the width of each avoided crossing is small compared to the spacing between modes. In other words, when many core modes are included, strong f-mode mixing can occur at multiple values of the planetary spin. Since the width of each strong mixing region is rather small, the chance of finding a core mode strongly mixed with the f-mode is also very small. A fine tuning in the spin frequency is required in order to realize the observed fine "splitting" of f-modes in Saturn.

\section{Effect of Oscillation Modes on Saturn's Rings}

Given a rotationally modified mode eigenfunction, we may calculate the gravitational potential perturbation it produces exterior to the planet. For a mode composed of a single spherical harmonic of angular degree $l$ and azimuthal number $m$, the potential produced outside the planet is

$$
\delta \Phi_{\alpha}(\mathbf{r}, t)=A_{\alpha} \delta \Phi_{\alpha}(R)\left(\frac{R}{r}\right)^{l+1} Y_{l m}(\theta, \phi) e^{i \sigma_{\alpha} t},
$$


where $\delta \Phi_{\alpha}(R)$ is the potential perturbation at the surface of the planet, calculated from our normalized mode eigenfunction (equation 7), $A_{\alpha}$ is the (unspecified) amplitude of the mode, and $\sigma_{\alpha}$ is the mode frequency in the inertial frame,

$$
\sigma_{\alpha}=\omega_{\alpha}-m \Omega_{s}
$$

This compact expression is modified in the presence of rotational mixing because each mode acquires contributions from multiple angular degrees $l$ (although $m$ remains a good "quantum" number). The potential produced by a rotationally modified mode is then

$$
\delta \Phi_{\alpha}(\mathbf{r}, t)=A_{\alpha} e^{i \sigma_{\alpha} t} \sum_{\beta} a_{\beta}^{(\alpha)} \delta \Phi_{\beta}(R)\left(\frac{R}{r}\right)^{l+1} Y_{l m}(\theta, \phi),
$$

where $a_{\beta}^{(\alpha)}$ is the projection of the rotationally modified mode onto an original mode $\beta$ (determined via the method in Appendix B, and the sum is over all original modes that contribute to the modified mode eigenfunction. For each term in the sum, the value of $l$ corresponds to the mode $\beta$.

We are interested in the value of the potential $\delta \Phi_{\alpha}$ in the plane of the rings $(\theta=\pi / 2)$ at the location of the outer Lindblad resonance. The resonance occurs where the forcing frequency experienced by a particle is equal to the local epicyclic frequency $\kappa$, i.e., where

$$
m\left(\Omega-\Omega_{p}\right)=\kappa,
$$

with $\Omega$ the local orbital frequency and $\Omega_{p}=-\sigma_{\alpha} / m$ the mode pattern frequency. The orbital and epicyclic frequencies are not exactly equal because of the oblateness of Saturn, which causes the two frequencies to differ by a factor $\sim 10^{-2}$ in the C-ring. Here we set them equal because we are concerned primarily with the strength of the potential (see Appenix Appendix D for a more accurate description). Then the resonant location is

$$
r_{L} \simeq\left[\frac{(1-m)^{2} G M}{\sigma_{\alpha}^{2}}\right]^{1 / 3}
$$

and the gravitational potential at the resonant location in the ring is

$$
\delta \Phi_{\alpha}\left(r_{L}\right) \simeq A_{\alpha} e^{i \sigma_{\alpha} t+m \phi} \sum_{\beta} a_{\beta}^{(\alpha)} Y_{l m}(\pi / 2,0)\left[\frac{(1-m)^{2}}{\sigma_{\alpha}^{2}} \frac{G M}{R^{3}}\right]^{-(l+1) / 3} \delta \Phi_{\beta}(R) .
$$

The effective potential driving waves at the Lindblad resonance is (Goldreich \& Tremaine 1979),

$$
\begin{aligned}
\Psi_{\alpha}\left(r_{L}\right) & =\left[\frac{d}{d \ln r}+\frac{2 m \Omega}{\sigma_{\alpha}+m \Omega}\right] \delta \Phi_{\alpha}\left(r_{L}\right) \\
& \simeq A_{\alpha} e^{i \sigma_{\alpha} t+m \phi} \sum_{\beta} a_{\beta}^{(\alpha)} W_{\beta} \delta \Phi_{\beta}(R)
\end{aligned}
$$

where the dimensionless constant

$$
W_{\beta}=(2 m-l-1) Y_{l m}(\pi / 2,0)\left[\frac{(1-m)^{2}}{\sigma_{\alpha}^{2}} \frac{G M}{R^{3}}\right]^{-(l+1) / 3} .
$$

The surface density variation and the associated optical depth variation produced near the Lindblad resonance are calculated in Appendix D.

The mode amplitudes $A_{\alpha}$ are unknown. To estimate them, we assume that the most prominent $|m|=3$ wave measured by HN13 is produced by an unmixed f-mode with frequency $\omega_{3}$, and we calculate the mode amplitude $A_{3}$ required to generate the observed optical depth variation in the ring (see Appendix D). We then assume the modes follow energy equipartition, such that their amplitudes satisfy $\omega_{\alpha}^{2}\left|A_{\alpha}\right|^{2}=\omega_{3}^{2}\left|A_{3}\right|^{2}{ }^{11}$

\footnotetext{
${ }^{11}$ Although energy equipartition may not apply to all modes, it is reasonable for our purpose since the modes of interest have similar frequencies $\omega_{\alpha}$ and angular degrees $l$.
} 
The typical mode amplitudes required to produce the observed fluctuations are of order $|A| \sim 10^{-9}$, resulting in radial surface displacements of order $\xi_{r}(R) \sim 30 \mathrm{~cm}$ for $\mathrm{f}$-modes. These amplitudes are similar to those claimed to be observed by Gaulme et al. (2011) in Jupiter and approximately what we might expect if the modes are stochastically excited via convective turbulence, analogous to the excitation of solar p-modes (see discussion in Marley \& Porco 1993). However, the detailed dynamics of mode excitation are beyond the scope of this paper.

\subsection{Comparison with Observations}

Figures 10,12 show the pattern frequencies $\Omega_{p}$ (and the associated Lindblad radii $r_{L}$, calculated via equation D.3) and the optical depth variations $\delta \tau$ (see equation D.6) produced by modes in our planetary models with different values of the shear modulus. We have also plotted the pattern frequencies and optical depth variations of the observed waves in the C-ring as tabulated by HN13.

We begin by examining the mode pattern frequencies. In each model, the pattern speed of the $l=-m$ f-mode in the non-rotating models is consistently too large. Adding the Coriolis force lowers the pattern frequency of the modified "f"-mode (i.e., the lowest frequency mode with a large value of $\delta \Phi_{\alpha}$ ), moving it closer to the observed pattern frequencies, although the predicted frequencies remain too high. Adding second-order rotational effects is expected to lower the predicted frequencies further, making them more consistent with observations. Obviously, with our simple planetary models, we should not expect exact agreement. Nonetheless, the close proximity of the predicted and observed pattern frequencies of the "f"modes is strong evidence that the observed waves are generated by Saturn's "f"-modes.

We can use Figures 10 -12 to understand the qualitative effects of rotationally-induced mode mixing. In the absence of rotation, the spectrum of modes with significant gravitational perturbations is sparse, and is due almost entirely to f-modes and low-order p-modes. However, as the rotation rate increases, a core mode well-separated in frequency from the f-mode obtains a potential perturbation

$$
\delta \Phi_{\alpha} \approx \frac{\left|C_{\mathrm{f} \alpha}\right|}{\bar{\omega}_{\mathrm{f}}-\bar{\omega}_{\alpha}} \delta \Phi_{\mathrm{f}}
$$

where $\bar{\omega}_{\mathrm{f}}$ and $\bar{\omega}_{\alpha}$ are the rotationally modified f-mode and core mode frequency, and $\left|C_{\mathrm{f} \alpha}\right|$ is their coupling coefficient (equation B.17). Consequently, many modes that had miniscule potential perturbations in the non-rotating model (and thus fall below the plotted range of Figures 10 12 obtain larger potential perturbations in the presence of rotation (although typically still orders of magnitude smaller than the f-mode), causing the mode frequency spectrum for rotating models to appear much denser in Figures 10

A mode nearly degenerate with the f-mode will obtain a potential perturbation $\delta \Phi_{\alpha} \simeq \delta \Phi_{\mathrm{f}} / \sqrt{2}$, although in both modes are really hybrid f-modes. In this case, the two modes are split in frequency by $\approx 2\left|C_{\mathrm{f} \alpha}\right|$. Our results suggest that such exact degeneracies are rare in our planetary models, as described in Section 4.3. To achieve significant mixing, the frequency separation between a core mode and f-mode, $\left|\Delta_{\alpha \alpha^{\prime}}\right|$, must be of order $\left|C_{\mathrm{f} \alpha}\right|$. However, in our models, the typical frequency separations $\left|\Delta_{\alpha \alpha^{\prime}}\right|$ between core modes are much larger than typical values of $\left|C_{\mathrm{f} \alpha}\right|$. Thus, the chances of a near degeneracy between a core mode and f-mode are small. Such degeneracies are possible (see e.g., the middle panel of Figure 10), but usually require fine-tuning of model parameters (such as the spin). Since frequency splitting is observed by HN13 for both $m=-2$ and $m=-3$ modes, we conclude that it is unlikely to be produced solely by Coriolis coupling between core modes and f-modes.

Finally, we examine the influence of the value of the shear modulus in the core. If Saturn's solid core has a shear modulus $\mu \gtrsim 8 \mathrm{GPa}$ (Figure 12), then the core shear modes have frequencies that are typically larger than the f-mode. Also, the core mode spectrum is sparse, making it very unlikely for core modes to mix strongly with the f-mode. Therefore, if $\mu \gtrsim 8 \mathrm{GPa}$, the observed waves in Saturn's rings cannot be generated by the addition of elastic core oscillation modes for reasonable Saturnian models. On the other hand, if the shear modulus is $\mu \lesssim 1.6 \mathrm{GPa}$ (Figure 10 , there may exist many oscillation modes with frequencies in the vicinity of the f-mode, allowing for the possibility of strong mode mixing. However, a smaller shear modulus also decreases the coupling coefficients, requiring a higher degree of degeneracy $\left(\operatorname{smaller}\left|\Delta_{\alpha \alpha^{\prime}}\right|\right.$ ) for efficient mode mixing to occur. 

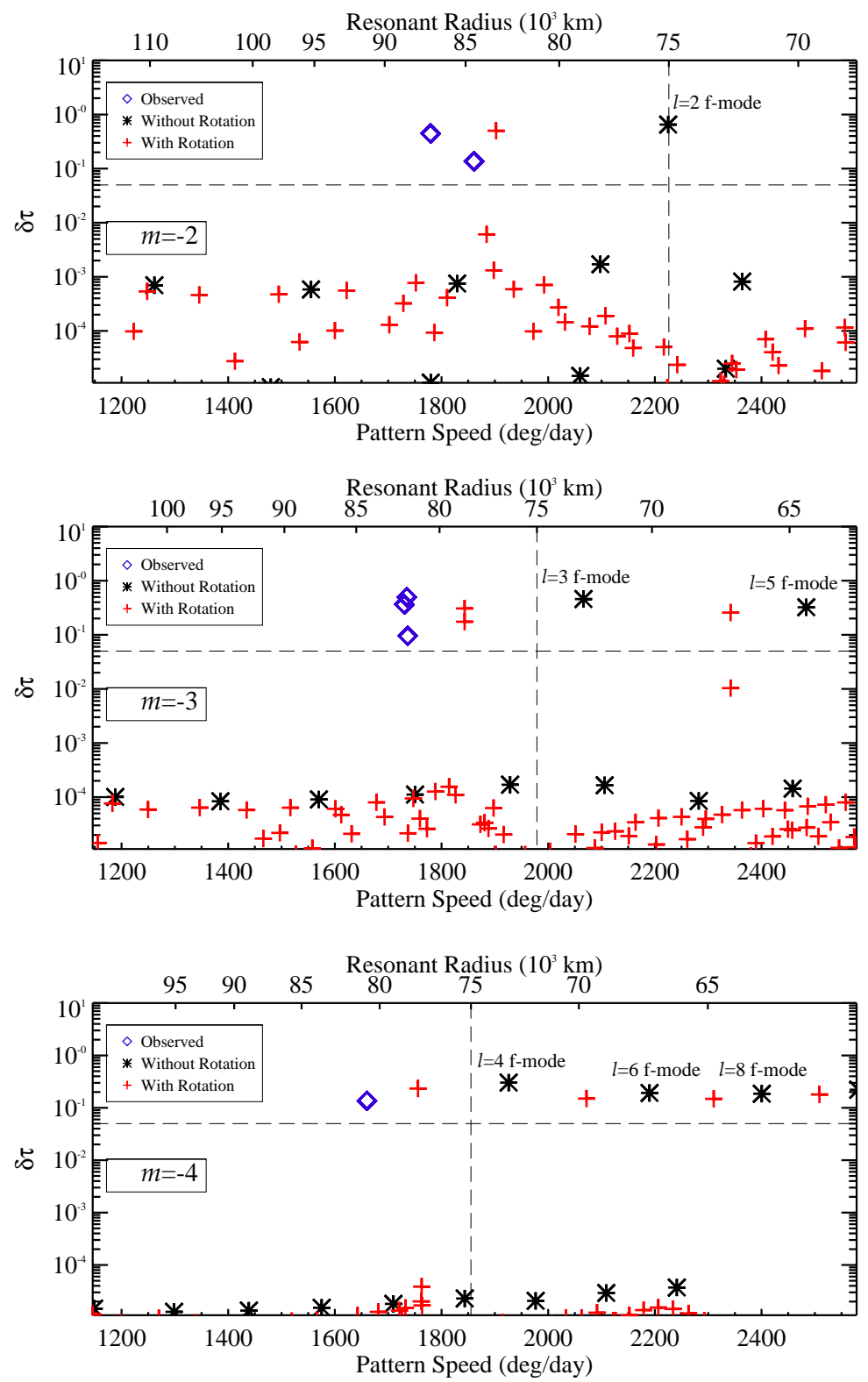

Figure 10: The predicted pattern frequencies $\sigma_{\alpha} /|m|$ and optical depth variations produced by waves at Lindblad resonances for the oscillation modes in our Saturn models, with $m=-2$ (top), $m=-3$ (middle), and $m=-4$ (bottom). We have plotted modes of non-rotating models (black asterisks), models rotating uniformly at Saturn's observed rotation frequency (red pluses), and the observed waves (blue diamonds) from HN13. These plots are made for a Saturn model with $n=1, R_{c}=0.25, D=4$, and $\mu=1.6 \mathrm{GPa}$. The spin frequency has been slightly tuned $\left(\Omega_{s} / \Omega_{s, o}=0.97\right)$ to produce two strongly mixed modes near the f-mode in the middle panel. The vertical dashed line indicates the approximate inner edge of the C-ring, while the horizontal dashed line is the approximate minimum observable optical depth variation. Therefore, we should only expect to observe waves in the top-left corner of the figure. In the non-rotating models, the f-modes are the black asterisks in the top right, while the row of asterisks below them are the $l=|m|$ s-modes. 

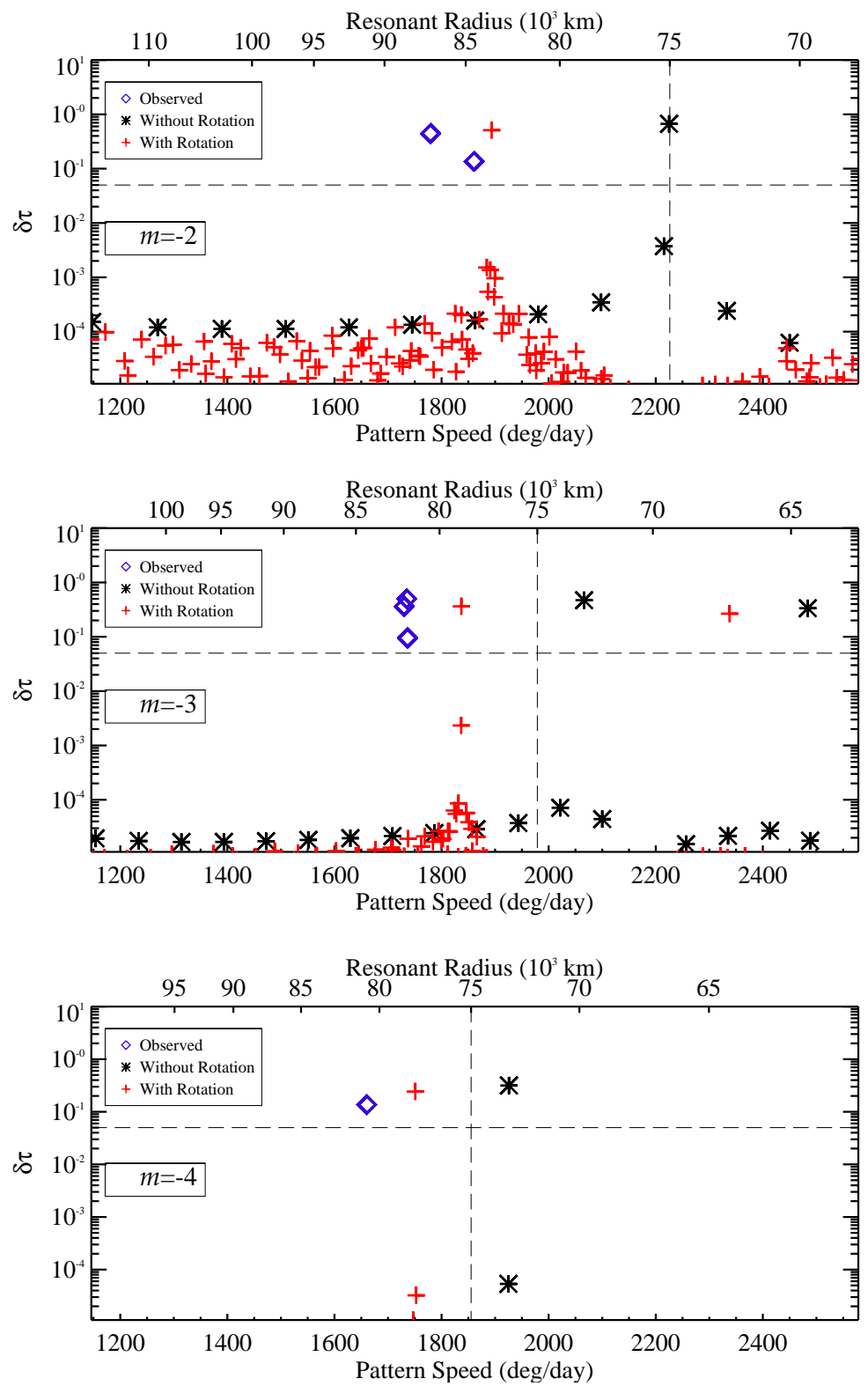

Figure 11: Same as Figure 10 but for a Saturn model with $\mu=0.32 \mathrm{GPa}$. 

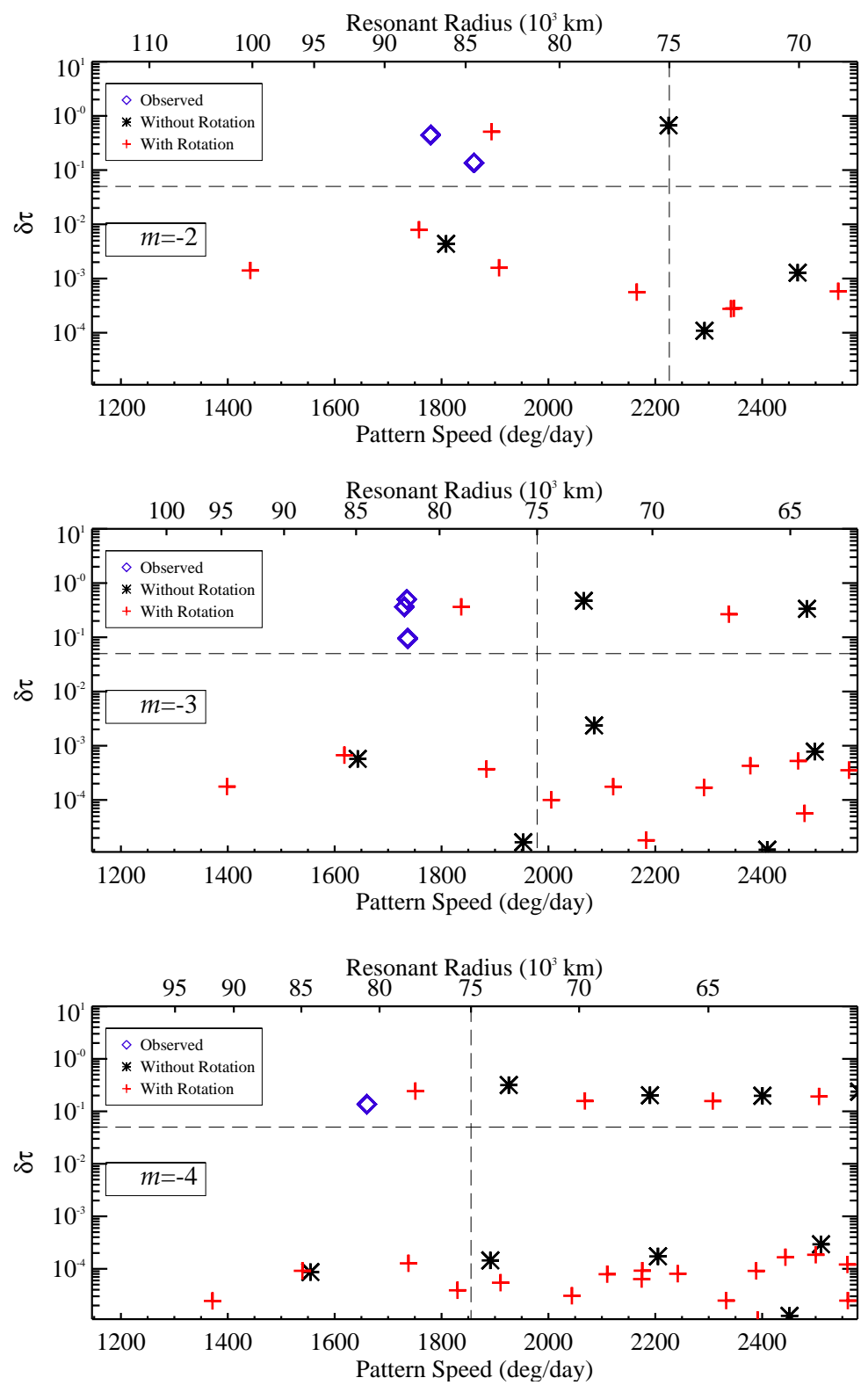

Figure 12: Same as Figure 10 but for a Saturn model $\mu=8 \mathrm{GPa}$. 


\section{Discussion and Conclusions}

We have examined the influence of a solid core on the oscillation mode spectrum of giant planets. In our planetary models, the rigidity of the core has a rather small effect on the frequencies of f-modes or low order p-modes, even for the largest core size $\left(R_{c}=0.25 R\right)$ and the range of shear modulus $\mu$ considered in this paper: the mode frequencies change change by less than 0.2 percent. However, the addition of a solid core adds two new branches of oscillation modes: the spheroidal and toroidal core modes, whose restoring force is the elastic shear force of the solid material. The frequency spectrum of these modes is dependent primarily on the core radius $R_{c}$ and shear modulus $\mu$, with larger and less rigid (small $\mu$ ) cores supporting dense spectra of low frequency shear modes. In the absence of rotation, these modes are almost completely confined to the core, and they produce negligible displacements or gravitational perturbations at the surface of the planet.

We have also examined the influence of the Coriolis force on the mode frequencies and eigenfunctions. In addition to changing the mode frequencies, the Coriolis force also induces mixing between oscillation modes of equal azimuthal number $m$. Spheroidal modes of angular degree $l$ mix with one another, as do toroidal modes. Furthermore, spheroidal modes of angular degree $l$ mix with toroidal modes of angular degree $l \pm 1$, allowing for chains of mode mixing extending to arbitrary values of $l$. Mode mixing is strongest when the rotationally modified frequencies of two modes are nearly degenerate with one another. If the value of the core shear modulus $\mu$ is small $(\mu \lesssim 3 \mathrm{GPa})$ but finite, the spectrum of core oscillation modes is dense near the f-mode. Therefore, one or multiple core modes may be nearly degenerate with the f-mode and mix strongly with it. These strongly mixed modes would manifest themselves as hybrid " $\mathrm{f}$ "-modes, with nearly equal frequencies and similar mode eigenfunctions.

One of the objectives of this investigation is to compare our planetary oscillation calculations with the results of HN13, who have measured the pattern numbers and frequencies of waves in Saturn's C ring that appear to be excited by Saturn's oscillation modes. As speculated by HN13 and originally proposed by Marley \& Porco (1993), we find that the pattern frequencies and azimuthal numbers associated with individual waves in Saturn's rings are consistent with being excited by Saturn's prograde sectoral $(l=-m)$ f-modes. It is possible that with more realistic planetary models and including higher order spin affects, the measured frequencies of these modes can be used to constrain Saturn's interior structure.

HN13's observations of multiple wave trains, finely spaced in frequency but with identical $m$, seem to indicate the existence of multiple oscillation modes with frequencies near the frequencies of Saturn's fmodes. We have shown that the rotation-induced mixing between shear modes in Saturn's core and f-modes in Saturn's fluid envelope can in principle lead to such finely spaced frequency splitting. Our theory has a number of notable features when comparing with the observations:

(i) Our theory explains why the waves observed by HN13 all lie close to the predicted locations of resonances with Saturn's f-modes. We have shown that only core modes very near in frequency to the f-mode will mix appreciably with it. Therefore, only these modes will obtain large enough gravitational potential perturbations to produce observable disturbances in Saturn's rings.

(ii) Our theory can in principle account for the relative magnitude of the observed frequency splittings (larger splitting for the $|m|=2$ modes, finer splittings for the $|m|=3$ modes, and no observable splitting for the $|m|=4$ mode). Compared to higher degree modes, the $l=2,|m|=2$ f-mode penetrates deeper into the planet. It has more inertia in the core, and thus mixes more strongly with core modes, as evidenced by its larger rotational coupling coefficients $\left|C_{\mathrm{f} \alpha}\right|$ with core modes. Therefore we should expect that modes that mix strongly with the $l=2 \mathrm{f}$-mode to have larger frequency separations from it than the modes that mix with the $l=3,|m|=3 \mathrm{f}$-mode or the $l=4,|m|=4 \mathrm{f}$-mode.

(iii) HN13's observations show that the finely spaced waves in Saturn's ring have different optical depth variations. In our theory, modes nearly degenerate with the f-mode may obtain potential perturbations comparable to (but less than) that of the unperturbed f-mode, with the precise values determined by the rotational coupling coefficient and degree of frequency detuning.

Despite these positive features, our theory of rotation-induced mode mixing suffers a serious fine-tuning problem when explaining the HN13 observations. Our calculations indicate that f-modes mix with core shear modes efficiently only when their frequency differences are very small. Such small frequency detunings are 
unlikely to occur unless the planetary model (including the rotation rate) is fine-tuned to produce a core mode nearly degenerate with the f-mode. Even with a finely tuned model, it is difficult to simultaneously achieve the observed $|m|=2$ and $|m|=3$ frequency splittings. Another concern is that a very small value of the shear modulus of the solid core $(\mu \lesssim 3 \mathrm{GPa})$ is required for our mechanism (in order to produce a dense spectrum of core modes near the f-mode frequency). In comparison, the shear modulus of Earth's core is $\mu \approx 100 \mathrm{GPa}$. (Laio et al. 2000), and the pressure in Saturn's core is of order $P \sim 2$ TPa. Although such a small value of the shear modulus may seem unlikely, it may be produced by the high pressure superionic phase of ice (see references in Section 2.1) likely to exist in Saturn's core.

There are a number of effects or perturbations that we have neglected in our paper and are worthy of further study. In general, for any perturbation $\mathcal{P}$ to induce strong mixing between an f-mode and another mode $\alpha$, the mixing matrix element $\mathcal{P}_{\text {f } \alpha}$ must satisfy (see Section 4 )

$$
\left|\mathcal{P}_{\mathrm{f} \alpha}\right| \gtrsim\left|\bar{\omega}_{f}-\bar{\omega}_{\alpha}\right|
$$

where $\bar{\omega}_{f}$ and $\bar{\omega}_{\alpha}$ are the mode frequencies (including the "self"-correction due the perturbation).

(i) We have already noted (see Section 1) that higher-order rotational effects (including the rotational distortion of the planet) can significantly change the f-mode frequency. This self-correction is of order $\sim$ $\Omega_{s}^{2} / \omega_{\mathrm{f}}$, and should be included when making detailed predictions of f-mode frequencies in different planetary models. The related mixing element $\left|\mathcal{P}_{\mathrm{f} \alpha}\right|$ is of the same order or less than $\Omega_{s}^{2} / \omega_{f}$, but nevertheless can be much larger compared to $\left|C_{\mathrm{f} \alpha}\right|$ associated with the Coriolis term. This may help alleviate the fine-tuning problem.

(ii) Differential rotation and other internal flows (such as that associated with slow convective motion) can also change mode frequencies, by the amount $\sim \Delta \Omega_{d}$ (differential rotation) or $\sim V_{\text {bulk }} / R$ (for bulk flow speed of order $\left.V_{\text {bulk }}\right)$. The related mixing element is of the same order or less. Since the observed fine splittings in HN13 are a few percent or less, these effects cannot be neglected.

(iii) Internal planetary magnetic fields can change the f-mode frequency by the amount $\sim\left(v_{A} / R\right)^{2} / \omega_{\mathrm{f}}$, where $v_{A}=\sqrt{B^{2} / 4 \pi \rho}$ is the charateristic Alfven speed. The fractional correction, $\sim\left(v_{A} / R \omega_{\mathrm{f}}\right)^{2}$ is less than $10^{-10}$ even for an extremely strong $(B \sim 1 \mathrm{kG})$ internal field. This effect may be neglected.

(iv) In this paper we have focused on f-mode mixing with shear modes in the core. But there may be other types of modes that can participate in the mixing. We have noted before (Section 1) that inertial modes or Rossby modes cannot directly mix with the f-mode because of their frequency mismatch. But they may be involved in secondary mixing in a multiple-mode mixing system (see Section 4.3). Also, in the presence of stratification of heavy elements (see Leconte \& Chabrier 2012), the fluid envelope may support gravity modes. These new modes can increase the "phase space" of mixing with f-modes, allowing them to be observed indirectly.

Overall, it remains unclear at present whether the finely spaced density waves observed in Saturn's Cring can be naturally produced by the simultaneous presence of finely split oscillation modes inside Saturn. In this regard, we should keep in mind the possibility that the observed waves are produced at different times, or the different wave frequencies reflect secular changes in the mode properties (such as amplitude and frequency) or internal properties (such as differential rotation) of the planet.

\section{Acknowledgments}

We thank Matt Hedman and Phil Nicholson for extensive discussions regarding the Cassini data used to measure the properties of the waves in Saturn's rings. This work has been supported in part by NSF grants AST-1008245, 1211061, NASA grants NNX12AF85G and NNX10AP19G.

\section{References}

[1] Alterman, Z., Jarosch, H., Pekeris, C., 1959, Proc. of the Royal Soc. of London, 252, 80

[2] Asahara, Y., Hirose, K., Ohishi, Y., Hirao, N., Murakami, M., 2010, Earth and Plan. Sci. Let., 299,474

[3] Baillie, K., Colwell, J., Lissauer, J., Esposito, L., Sremcevic, M., 2011, Icarus, 216, 292 
[4] Cavazzoni, C., Chiarotti, G., Scandalo, S., Tosatti, E., Bernasconi, M., Parrinello, M., 1999, Science, 283, 44

[5] Chaplin, W., Miglio, A., 2013, Ann. Rev. Ast. Astro, 51

[6] Colwell, J. E., Nicholson, P. D., Tiscareno, M. S., Murray, C. D., French, R. G., Marouf, E. A., 2009, Saturn from Cassini-Huygens, ed. Dougherty, M. K., Esposito, L. W., \& Krimigis, S. M. Cambridge U., 375-412

[7] Crossley, D., 1975, Geophys. J. R. Astr. Soc., 41, 153

[8] Dyson, J., Schutz, B., 1979, Roy Soc. Proc. Series A, 368, 389

[9] Dahlen, F., Tromp, J., 1998, Theoretical Global Seismology, Princeton University Press

[10] Fortney, J., Baraffe, I., Militzer, B., 2009, arXiv:0911.3154 1

[11] French, M., Mattsson, T., Nettelmann, N., Redmer, R., 2009, Phys. Rev. B., 79, 054107

[12] Gaulme, P, Schmider, F., Gay, J., Guillot, T., Jacob, C., 2011 A\&A, 531, 104

13] Greenberg, R., Brahic, A., 1984, Planetary Rings, University of Arizona Press

[14] Guillot, T., 2005, Annu. Rev. Earth Planet. Sci., 33, 493

[15] Hansen, C., Van Horn, H., 1979, ApJ, 233, 253

[16] Hedman, M., Nicholson, P., 2013, arXiv:1304.3735

[17] Hermann, A., Ashcroft, N., Hoffmann, R., Proc. of the Nat. Acad. of Sci., 2012, 109, 745

[18] Laio, A., Bernard, S., Chiarotti, G., Scandolo, S., Tosatti, E., 2000, Science, 287, 1027

[19] Le Bihan, B., Burrows, A., ApJ, 764, 18

[20] Leconte, J., Chabrier, G., 2012, A\&A 540, A20

[21] Marley, M., 1991, Icarus, 94, 420

[22] Marley, M., Porco, C., 1993, Icarus, 106, 508

[23] McDermott, P., Van Horn, H., Hansen, C., 1988, 325, 725

[24] Militzer, B., Hubbard, W., Vorberger, J., Tamblyn, I., Bonev, S. A., 2008, ApJ, 688, L45

[25] Militzer, B., Wilson, H., 2010, PRL 105, 195701

[26] Militzer, B., 2012, Int. Jour. of Quant. Chem., 112, 314

[27] Montgomery, M., Winget, D., 1999, ApJ, 526, 976

[28] Press, W.H., Teukolsky, S.A., Vetterling, W.T., Flannery, B.P., 1998, Numerical Recipes (Cambridge Univ. Press)

[29] Rosen, P. A., Tyler, G. L., Marouf, E. A., \& Lissauer, J. J., 1991, Icarus, 93, 25

[30] Vorontsov, S., Zharkov, V., 1981, Atron. Zh., 58, 1101

[31] Vorontsov, S., 1981, Atron. Zh., 58, 1275

[32] Wang, Y., Liu, H., Lv, J., Zhu, L., Wang, H., Ma, Y., 2011, Nature Comm., 2, 563

[33] Wilson, H., Militzer, 2012, ApJ, 745, 54

[34] Wu, Y., 2005, ApJ, 635, 674

\section{Appendix A. Elastic Oscillation Equations}

In this work we consider purely adiabatic and elastic oscillations, i.e., we neglect damping produced by non-adiabaticity and anelasticity. Under these approximations, the elastic forces act like a spring, which is characterized by the stress tensor

$$
\boldsymbol{\sigma}=K(\boldsymbol{\nabla} \cdot \boldsymbol{\xi}) \mathbf{I}+2 \mu \mathbf{s}
$$

Here, $K$ is the adiabatic bulk modulus, related to the pressure $P$ via $K=\Gamma_{1} P$, with $\Gamma_{1}$ the adiabatic index of the material. Also, $\mu$ is the shear modulus, $\boldsymbol{\xi}$ is the vector displacement, $\mathbf{I}$ is the identity matrix, and the deviatoric strain tensor $\mathbf{s}$ is defined as

$$
\mathbf{s} \equiv \frac{1}{2}\left[\boldsymbol{\nabla} \boldsymbol{\xi}+(\boldsymbol{\nabla} \boldsymbol{\xi})^{T}\right]-\frac{1}{3}(\boldsymbol{\nabla} \cdot \boldsymbol{\xi}) \mathbf{I}
$$

We assume that the unperturbed state of the planet has zero elastic stress. The perturbed hydroelastic oscillation equation reads

$$
\frac{\partial^{2} \boldsymbol{\xi}}{\partial t^{2}}=\frac{\delta \rho}{\rho^{2}} \nabla P-\frac{1}{\rho} \nabla \delta P-\nabla \delta \Phi+\delta \mathbf{f}_{e},
$$

where the perturbed elastic force $\delta \mathbf{f}_{e}$ is

$$
\begin{aligned}
\delta \mathbf{f}_{e} & =\frac{2}{\rho} \boldsymbol{\nabla} \cdot(\mu \mathbf{s}) \\
& =\frac{2}{\rho} \boldsymbol{\nabla} \mu \cdot \mathbf{s}+\frac{\mu}{\rho}\left(\nabla^{2} \boldsymbol{\xi}+\frac{1}{3} \boldsymbol{\nabla}(\boldsymbol{\nabla} \cdot \boldsymbol{\xi})\right)
\end{aligned}
$$


We decompose all variables into spherical harmonics, or spherical vector harmonics where appropriate:

$$
\begin{aligned}
\boldsymbol{\xi}(\mathbf{r}, t) & =\sum_{l, m}\left[U_{l}(r) Y_{l m} \hat{\mathbf{r}}+V_{l}(r) r \nabla Y_{l m}+W_{l}(r) \boldsymbol{\nabla} \times\left(\mathbf{r} Y_{l m}\right)\right] e^{i \omega t}, \\
\delta \Phi(\mathbf{r}, t) & =\sum_{l, m}\left[\delta \Phi_{l}(r) Y_{l m}\right] e^{i \omega t}
\end{aligned}
$$

and similarly for $\delta \rho$ and $\delta P$. Then the equations become separated such that only r-dependence is left, and the equations for different harmonics are self-contained. From now on we will drop the subscript $l$ and consider the equations for a single harmonic. Explicitly, the displacement vector becomes:

$$
\begin{aligned}
\xi_{r} & =U(r) Y_{l m} \\
\xi_{\theta} & =V(r) \frac{\partial Y_{l m}}{\partial \theta}+\frac{W(r)}{\sin \theta} \frac{\partial Y_{l m}}{\partial \phi} \\
\xi_{\phi} & =\frac{V(r)}{\sin \theta} \frac{\partial Y_{l m}}{\partial \phi}-W(r) \frac{\partial Y_{l m}}{\partial \theta} .
\end{aligned}
$$

In addition, there is the Poisson equation for $\delta \Phi$ :

$$
\nabla^{2} \delta \Phi=4 \pi G \delta \rho .
$$

We also define the function $\alpha(r)$ via

$$
\alpha Y_{l m}=\boldsymbol{\nabla} \cdot \boldsymbol{\xi}=\left[\dot{U}+\frac{2}{r} U-\frac{l(l+1)}{r} V\right] Y_{l m},
$$

where the dot denotes radial derivative. The continuity equation gives

$$
\begin{aligned}
\delta \rho & =-\boldsymbol{\xi} \cdot \nabla \rho-\rho \boldsymbol{\nabla} \cdot \boldsymbol{\xi} \\
& =-(U \dot{\rho}+\rho \alpha) Y_{l m} .
\end{aligned}
$$

Equation (A.3) represents three 2nd-order ordinary differential equations for $U, V, W$, while equation A.7) is a 2nd-order ODE for $\delta \Phi$. We wish to transform these four second-order ODEs into eight first-order ODEs. First we define a new quantity, the Lagrangian traction $\mathbf{T} \equiv \hat{\mathbf{r}} \cdot \Delta \boldsymbol{\sigma}$, where $\Delta \sigma_{i j}=K \alpha \delta_{i j}+2 \mu s_{i j}$. Then we have

$$
\begin{aligned}
\mathbf{T} & =\hat{\mathbf{r}} \cdot \Delta \boldsymbol{\sigma} \\
& =T_{r}(r) Y_{l m} \hat{\mathbf{r}}+T_{\perp}(r) r \boldsymbol{\nabla} Y_{l m}+T_{t}(r) \boldsymbol{\nabla} \times\left(\mathbf{r} Y_{l m}\right) \\
& =\left(\left[K-\frac{2}{3} \mu\right] \alpha+2 \mu \dot{U}\right) Y_{l m} \hat{\mathbf{r}} \\
& +\mu\left\{\left(\frac{U}{r}+\dot{V}-\frac{V}{r}\right) \frac{\partial Y_{l m}}{\partial \theta}+\left(\dot{W}-\frac{W}{r}\right) \frac{1}{\sin \theta} \frac{\partial Y_{l m}}{\partial \phi}\right\} \hat{\theta} \\
& +\mu\left\{\left(\frac{U}{r}+\dot{V}-\frac{V}{r}\right) \frac{1}{\sin \theta} \frac{\partial Y_{l m}}{\partial \phi}-\left(\dot{W}-\frac{W}{r}\right) \frac{\partial Y_{l m}}{\partial \theta}\right\} \hat{\phi} .
\end{aligned}
$$


We now define 8 new variables as follows:

$$
\begin{aligned}
& y_{1}=U, \\
& y_{2} \equiv T_{r}=\left(K-\frac{2}{3} \mu\right) \alpha+2 \mu \dot{U}, \\
& y_{3}=V, \\
& y_{4} \equiv T_{\perp}=\mu\left(\frac{U}{r}+\dot{V}-\frac{V}{r}\right), \\
& y_{5}=\delta \Phi \\
& y_{6}=\dot{y}_{5}+4 \pi G \rho y_{1}, \\
& y_{7}=W \\
& y_{8} \equiv T_{t}=\mu\left(\dot{W}-\frac{W}{r}\right) .
\end{aligned}
$$

Note that $y_{1}, y_{3}, y_{7}$ are the displacement components, while $y_{2}, y_{4}, y_{8}$ are the Lagrangian tractions. $y_{6}$ is the Lagrangian gravitational attraction, chosen as a variable for convenience when applying boundary conditions (see section Appendix A.1). The final oscillation equations are:

$$
\begin{aligned}
\dot{y}_{1} & =-\frac{2\left(K-\frac{2}{3} \mu\right)}{\left(K+\frac{4}{3} \mu\right)} \frac{y_{1}}{r}+\frac{1}{\left(K+\frac{4}{3} \mu\right)} y_{2}+\frac{l(l+1)\left(K-\frac{2}{3} \mu\right)}{\left(K+\frac{4}{3} \mu\right)} \frac{y_{3}}{r}, \\
\dot{y}_{2} & =\left[-4 \rho g-\omega^{2} \rho r+\frac{12 \mu K}{\left(K+\frac{4}{3} \mu\right) r}\right] \frac{y_{1}}{r}-\frac{4 \mu}{\left(K+\frac{4}{3} \mu\right)} \frac{y_{2}}{r} \\
& +l(l+1)\left[\rho g-\frac{6 \mu K}{\left(K+\frac{4}{3} \mu\right) r}\right] \frac{y_{3}}{r}+l(l+1) \frac{y_{4}}{r}+\rho y_{6}, \\
\dot{y}_{3} & =-\frac{y_{1}}{r}+\frac{y_{3}}{r}+\frac{y_{4}}{\mu}, \\
\dot{y}_{4} & =\left[\rho g-\frac{6 K \mu}{\left(K+\frac{4}{3} \mu\right) r}\right] \frac{y_{1}}{r}-\frac{\left(K-\frac{2}{3} \mu\right)}{\left(K+\frac{4}{3} \mu\right)} \frac{y_{2}}{r} \\
& +\left\{-\omega^{2} \rho r+\frac{2 \mu\left[\left(K-\frac{2}{3} \mu\right)\left(2 l^{2}+2 l-1\right)+2 \mu\left(l^{2}+l-1\right)\right]}{\left(K+\frac{4}{3} \mu\right) r}\right\} \frac{y_{3}}{r} \\
& -3 \frac{y_{4}}{r}+\rho \frac{y_{5}}{r}, \\
\dot{y}_{5} & =-4 \pi G \rho y_{1}+y_{6}, \\
\dot{y}_{6} & =4 \pi G \rho l(l+1) \frac{y_{3}}{r}+\frac{l(l+1)}{r} \frac{y_{5}}{r}-\frac{2}{r} y_{6}, \\
\dot{y}_{7} & =\frac{y_{7}}{r}+\frac{y_{8}}{\mu}, \\
\dot{y}_{8} & =\left[-\omega^{2} \rho r+\frac{\mu\left(l^{2}+l-2\right)}{r}\right] \frac{y_{7}}{r}-\frac{3}{r} y_{8} .
\end{aligned}
$$

Note that $y_{7}$ and $y_{8}$ completely decouple from the other six equations. The first six equations are integrated to obtain the spheroidal modes, while the last two are integrated to obtain the toroidal modes.

In fluid regions where $\mu \rightarrow 0, y_{4} \rightarrow 0$ and $y_{3}$ is found from the algebraic relation

$$
y_{3}=\frac{1}{r \omega^{2}}\left(g y_{1}-\frac{1}{\rho} y_{2}+y_{5}\right) .
$$

Thus only four ODEs are needed for the fluid region. 


\section{Appendix A.1. Boundary Conditions}

The boundary conditions (BCs) for elastic oscillations can be found in Dahlen and Tromp (1998) and in Crossley (1975). For the spheroidal oscillations, the BCs at $r=0$ are, to lowest order in $r$,

$$
\begin{aligned}
& y_{1}=A r^{l-1}, \\
& y_{2}=2(l-1) \mu A r^{l-2}, \\
& y_{3}=\frac{A}{l} r^{l-1}, \\
& y_{4}=\frac{2(l-1) \mu A}{l} r^{l-2}, \\
& y_{5}=\left[-\frac{4 \pi G \rho A}{l}+\frac{B}{l}\right] r^{l} . \\
& y_{6}=B r^{l-1} .
\end{aligned}
$$

For $l=0, A=0$ and higher order terms in $r$ are needed to establish the BCs (see Crossley 1975). When $l=1$, the displacements are finite at the center of the planet. Note that for $l=2$, the values of the tractions $T_{r}$ and $T_{\perp}$ are finite, i.e., the core is undisplaced but is under stress. For $l>2$, the values of all perturbation variables are zero at the center of the planet. Equations (A.28)- A.33 comprise only two independent BCs (corresponding to the undetermined constants $A$ and $B$ ), and the additional inner BCs are trivially satisfied due to the form of the oscillation equations. In practice, for $l \geq 2$, we use the four inner BCs

$$
\begin{aligned}
& y_{1}=0, \\
& y_{2}=l y_{4}, \\
& y_{4}=0, \\
& y_{6}=0,
\end{aligned}
$$

although other choices are possible.

The three independent boundary conditions at the surface of the planet are

$$
\begin{aligned}
& y_{2}=0, \\
& y_{4}=0, \\
& y_{6}=-\frac{l+1}{R} y_{5} .
\end{aligned}
$$

Note that in the fluid envelope, the first of these conditions is equivalent to the boundary condition $\Delta P=0$ commonly used in asteroseismology. The condition on $y_{4}$ is trivially satisfied in the planetary model with a fluid envelope, since $y_{4}=0$ in fluid regions. In our calculations, we instead use the outer BCs involving $y_{2}$ and $y_{6}$, the four inner BCs listed above, and the jump conditions at core-envelope interface described below.

At the solid core-fluid envelope interface, the values of $y_{1}, y_{2}, y_{4}, y_{5}$, and $y_{6}$ must be continuous. There is no relation to determine the change in $y_{3}$ across the interface, and in general $y_{3}$ is discontinuous. Note that the continuity of $y_{6}$ across an interface implies a discontinuity in the gravitational perturbation, $\delta d \Phi / d r$. Also note that the continuity of $y_{4}$ implies that it is zero at a fluid-solid interface. For torsional oscillations of $l \geq 2$, the BCs are $y_{7}=0$ at $r=0$ and $y_{8}=0$ at $r=R$. At a fluid-solid interface, $y_{8}$ is continuous but $y_{7}$ is generally discontinuous.

Solving the eigensystem A.19)- A.26 for the eigenvalue $\omega$ also requires a normalization boundary condition. Typically, the normalization $y_{1}(R)=1$ is used, although the choice of normalization is entirely arbitrary. For oscillation modes in planets with a solid core and a fluid envelope, this condition is poorly suited for numerical computation. The reason is that p-modes confined to the fluid envelope have very large relative surface displacements, while shear modes confined to the solid core have very small relative surface displacements. For our models, we find the normalization condition $y_{3}=1$ just below the core-envelope boundary allows our code to quickly converge for both p-modes and shear modes. The eigenmodes are then renormalized via equation (7). 


\section{Appendix B. Solving for Rotationally Mixed Modes}

In the rotating frame of the planet, the perturbed momentum equation takes the form

$$
-\omega^{2} \boldsymbol{\xi}+2 i \omega \boldsymbol{\Omega}_{s} \times \boldsymbol{\xi}+\mathcal{H} \boldsymbol{\xi}=0,
$$

where $\mathcal{H} \boldsymbol{\xi}$ is given by the negative of the right hand side of equation $A .3$, and we have used $\boldsymbol{\xi} \propto e^{i \omega t}$. Let

$$
\boldsymbol{\xi}(\mathbf{r})=\sum_{\alpha} a_{\alpha} \boldsymbol{\xi}_{\alpha}(\mathbf{r}),
$$

where $\boldsymbol{\xi}_{\alpha}(\mathbf{r})$ satisfies

$$
-\omega_{\alpha}^{2} \boldsymbol{\xi}_{\alpha}+\mathcal{H} \boldsymbol{\xi}_{\alpha}=0 .
$$

Then we obtain

$$
\left(\omega_{\alpha}^{2}-\omega^{2}\right) a_{\alpha}+2 \omega \sum_{\beta} C_{\alpha \beta} a_{\beta}=0,
$$

where

$$
\begin{aligned}
C_{\alpha \beta} & =\left\langle\boldsymbol{\xi}_{\alpha} \mid i \boldsymbol{\Omega}_{s} \times \boldsymbol{\xi}_{\beta}\right\rangle \\
& =\int d V \rho \boldsymbol{\xi}_{\alpha}^{*} \cdot\left(i \boldsymbol{\Omega}_{s} \times \boldsymbol{\xi}_{\beta}\right) .
\end{aligned}
$$

Explicit expressions for the mixing coefficient $C_{\alpha \beta}$ are given in Appendix B.2

Equation (B.4) represents an eigensystem for the modified eigenvalues $\omega$, with each row in the matrix equation indexed by $\alpha$ and each column indexed by $\beta$. For each eigenvalue $\omega^{2}$, the complex components of the eigenvector $\mathbf{a}=\left\{a_{1}, \ldots a_{N}\right\}$ represent the projection of the new eigenmode onto each original mode $\beta$. In matrix form, equation $B .4$ is a quadratic eigenvalue problem:

$$
(\boldsymbol{\Omega}+2 \omega \mathbf{C}) \mathbf{a}=\omega^{2} \mathbf{I a},
$$

where $\boldsymbol{\Omega}=\operatorname{diag}\left\{\omega_{1}^{2}, \ldots, \omega_{N}^{2}\right\}, \mathbf{I}$ is the identity matrix, and

$$
\mathbf{C}=\left[\begin{array}{ccc}
C_{11} & \cdots & C_{1 N} \\
\vdots & \ddots & \vdots \\
C_{N 1} & \cdots & C_{N N}
\end{array}\right]
$$

The $N \times N$ eigenvalue problem of equation $(\mathrm{B} .6)$ is equivalent to the $2 N \times 2 N$ eigenvalue problem

$$
\left[\begin{array}{cc}
0 & \mathbf{I} \\
\mathbf{\Omega} & 2 \mathbf{C}
\end{array}\right]\left[\begin{array}{l}
\mathbf{a} \\
\mathbf{b}
\end{array}\right]=\omega\left[\begin{array}{l}
\mathbf{a} \\
\mathbf{b}
\end{array}\right],
$$

where $\mathbf{b}=\omega \mathbf{a}$. Equation $(\overline{B .8})$ is equivalent to the phase space mode expansion, where a mode $\mathbf{Z}$ of eigenvalue $\omega$ is defined by both its displacement and velocity vectors such that $\mathbf{Z}=[\mathbf{a}, \mathbf{b}]=[\mathbf{a}, \omega \mathbf{a}]$. Solving the eigensystem of equation (B.8) yields $2 N$ eigenvalues $\omega$. In the limit $\Omega_{s} \rightarrow 0$, the solutions come in pairs, $\omega_{\alpha}$ and $-\omega_{\alpha}$, representing the prograde and retrograde modes. Note that in this form, the matrix on the left hand side of equation (B.8) is not explicitly Hermitian, however, we show below that the system can be written in a Hermitian form.

We solve the eigensystem B.8 for the eigenvalues $\omega$ and the associated eigenfunctions a. Because only modes of the same $m$ are coupled (see Section Appendix B.2), we may solve equation B.8 separately for each value of $m$ we wish to consider. For negative $m$, the positive values of $\omega$ represent prograde modes, and the negative values of $\omega$ represent retrograde modes. In principle, we must include every oscillation mode (i.e., for a given $m$ we must include modes of all $l$ and $n$ ) in the eigensystem. In practice, we truncate the eigensystem at finite values of $l$ and $n$. To determine which modes to include, we can solve equation (B.8) for a limited set of modes near the $l=|m|$ f-modes, and then extend our calculations to larger values of $l$ and larger frequency ranges to see if the results change. For the planetary models, spin frequencies, and mode frequencies considered in this paper, we find that including only modes with $l \lesssim 14$ and $\omega_{l, n} / 3 \lesssim \omega_{f} \lesssim 3 \omega_{l, n}$ yields a good approximation. 


\section{Appendix B.1. Alternative Formalism}

The eigensystem equation (B.8) can be solved more elegantly, as shown in Dyson \& Schutz (1979) and DT98. We seek to solve the eigenvalue problem

$$
[\mathcal{H}+\omega \overline{\mathcal{C}}] \boldsymbol{\xi}=\omega^{2} \boldsymbol{\xi},
$$

where $\mathcal{H}$ has the same definition as above, and $\overline{\mathcal{C}}=2 i \boldsymbol{\Omega}_{s} \times$. Defining each eigenvector via its six-dimensional eigenfunction

$$
\mathbf{Z}=\left[\begin{array}{c}
\boldsymbol{\xi} \\
\omega \boldsymbol{\xi}
\end{array}\right]
$$

We rewrite equation $(B .9)$ as

$$
\left(\left[\begin{array}{cc}
0 & \mathcal{I} \\
\mathcal{H} & 0
\end{array}\right]+\left[\begin{array}{cc}
0 & 0 \\
0 & \overline{\mathcal{C}}
\end{array}\right]\right) \mathbf{Z}=\omega \mathbf{Z}
$$

where $\mathcal{I}$ is the identity operator. We decompose $\mathbf{Z}$ in terms of original eigenvectors,

$$
\mathbf{Z}=\sum_{\beta} a_{\beta} \mathbf{Z}_{\beta},
$$

where here the sum runs over the $2 N$ components $\beta$ (accounting for both negative and positive eigenfrequencies) because we have employed the phase space mode expansion, in contrast to the configuration space expansion used in equation B.12. Recalling that $\mathcal{H} \boldsymbol{\xi}_{\beta}=\omega_{\beta}^{2} \boldsymbol{\xi}_{\beta}$, equation B.11 becomes

$$
\sum_{\beta} \omega_{\beta} a_{\beta} \mathbf{Z}_{\beta}+\sum_{\beta}\left[\begin{array}{cc}
0 & 0 \\
0 & \overline{\mathcal{C}}
\end{array}\right] a_{\beta} \mathbf{Z}_{\beta}=\sum_{\beta} \omega a_{\beta} \mathbf{Z}_{\beta} .
$$

We now multiply by the auxiliary operator

$$
\mathcal{P}=\left[\begin{array}{cc}
\mathcal{H} & 0 \\
0 & \mathcal{I}
\end{array}\right],
$$

and take the inner product with $\mathbf{Z}_{\alpha}$ to obtain

$$
2 \omega_{\alpha}^{3} a_{\alpha}+2 \omega_{\alpha} \sum_{\beta} \omega_{\beta} a_{\beta} C_{\alpha \beta}=2 \omega_{\alpha}^{2} \omega a_{\alpha},
$$

where $C_{\alpha \beta}$ has the same definition as above. Defining $b_{\alpha}=\omega_{\alpha} a_{\alpha}$, we have

$$
\omega_{\alpha} b_{\alpha}+\sum_{\beta} C_{\alpha \beta} b_{\beta}=\omega b_{\beta} .
$$

Equation (B.16) represents a Hermitian eigensystem because $C_{\alpha \beta}=C_{\beta \alpha}^{*}$, therefore it is amenable to numerical matrix solving techniques. Once the eigenvectors $\mathbf{b}$ are determined, components of the desired eigenvector a are obtained by $a_{\beta}=b_{\beta} / \omega_{\beta}$.

\section{Appendix B.2. Rotational Mixing Coefficients}

The element $C_{\alpha \alpha^{\prime}}$ of the rotational coupling matrix is defined as

$$
C_{\alpha \alpha^{\prime}}=i \Omega_{s} \int d V \rho \boldsymbol{\xi}_{\alpha}^{*} \cdot\left(\hat{\mathbf{z}} \times \boldsymbol{\xi}_{\alpha^{\prime}}\right),
$$


where $\hat{\mathbf{z}}$ is the unit vector in the $z$ direction, and the integral is over the volume of the planet. Explicitly, the value of $C_{\alpha \alpha^{\prime}}$ is (see also Dahlen \& Tromp 1998)

$$
\begin{aligned}
C_{\alpha \alpha^{\prime}} & =m \Omega_{s} \delta_{l l^{\prime}} \delta_{m m^{\prime}} \int_{0}^{R} d r \rho r^{2}\left(U V^{\prime}+V U^{\prime}+V V^{\prime}+W W^{\prime}\right) \\
& -\frac{i \Omega_{s}}{2}\left(S_{l m} \delta_{l l^{\prime}+1}+S_{l^{\prime} m} \delta_{l l^{\prime}-1}\right) \delta_{m m^{\prime}} \\
& \times \int_{0}^{R} d r \rho r^{2}\left[\left(k_{l}^{2}-k_{l^{\prime}}^{2}-2\right) U W^{\prime}+\left(k_{l}^{2}-k_{l^{\prime}}^{2}+2\right) U^{\prime} W+\left(k_{l}^{2}+k_{l^{\prime}}^{2}-2\right)\left(V W^{\prime}-V^{\prime} W\right)\right],
\end{aligned}
$$

with $k_{l}^{2}=l(l+1)$ and

$$
S_{l m}=\left[\frac{(l+m)(l-m)}{(2 l+1)(2 l-1)}\right]^{1 / 2} .
$$

For compactness, we have dropped the $\alpha$ subscript on the right hand side of equation (B.18). The displacement functions $U, V$, and $W$ are defined in equation (A.6). The first integral in equation (B.18) accounts for spheroidal-spheroidal coupling and toroidal-toroidal mode coupling. For $\alpha=\alpha^{\prime}$, it reduces to the conventional rotational splitting parameter. The second integral in equation (B.18) accounts for spheroidal-toroidal mode coupling. Note the Hermitian nature of $C_{\alpha \alpha^{\prime}}$, i.e., $C_{\alpha \alpha^{\prime}}=C_{\alpha^{\prime} \alpha}^{*}$.

The value of $C_{\alpha \alpha^{\prime}}$ is zero unless the modes satisfy certain angular selection rules. In particular, only modes of equal azimuthal number $m=m^{\prime}$ can couple since the introduction of rotation does not break the axial symmetry of the problem. Similarly, spheroidal modes couple only to other spheroidal modes of equal angular degree such that $l=l^{\prime}$. The same is true for toroidal modes. However, spheroidal modes of degree $l$ may couple to toroidal modes of degree $l^{\prime}=l \pm 1$, and vice versa. Therefore, spheroidal modes of $Y_{l, m}$ are coupled to toroidal modes of $Y_{l+1, m}$, which in turn are coupled to spheroidal modes of $Y_{l+2, m}$, etc. Thus, the inclusion of the Coriolis forces introduces an infinite chain of coupling with modes of higher angular degree $l$.

\section{Appendix B.3. Mode Normalization}

The modes obtained by solving the eigensystem $(\mathrm{B} .8)$ or $\mathrm{B} .16$ must be appropriately normalized, because they are no longer orthonormal under equation (7). Instead, the modes satisfy the modified orthonormality condition

$$
\left\langle\left\langle\mathbf{Z}^{(\alpha)} \mid \mathbf{Z}^{\left(\alpha^{\prime}\right)}\right\rangle\right\rangle \equiv\left\langle\mathbf{Z}^{(\alpha)}|\mathcal{P}| \mathbf{Z}^{\left(\alpha^{\prime}\right)}\right\rangle=2\left(\omega^{(\alpha)}\right)^{2} \delta_{\alpha, \alpha^{\prime}},
$$

with $\mathcal{P}$ defined by equation (B.14), and the $(\alpha)$ superscript is the index of the rotationally modified mode. Recall that the decomposition of equation $(\mathrm{B} .12$ runs over the $2 N$ indices corresponding to $N$ mode pairs with opposite frequencies and identical eigenfunctions. The normalization condition (B.20) becomes

$$
\sum_{\beta}\left(\left|a_{\beta}^{(\alpha)}\right|^{2} \omega_{\beta}^{2}+a_{\beta}^{(\alpha)^{*}} a_{-\beta}^{(\alpha)} \omega_{-\beta}^{2}\right)+\omega_{\alpha}^{2} \sum_{\beta}\left(\left|a_{\alpha, \beta}\right|^{2}+a_{\beta}^{(\alpha)^{*}} a_{-\beta}^{(\alpha)}\right)=2 \omega_{\alpha}^{2},
$$

where the subscript $-\beta$ refers to the original mode with eigenfrequency of opposite sign. The sum in equation (B.21) over the $2 N$ modes $\beta$ can be written more simply as a sum over the $N$ modes with $\omega_{\beta}>0$ :

$$
\sum_{\omega_{\beta}>0} \frac{1}{2}\left[1+\frac{\omega_{\beta}^{2}}{\left(\omega^{(\alpha)}\right)^{2}}\right]\left|a_{\beta}^{(\alpha)}+a_{-\beta}^{(\alpha)}\right|^{2}=1
$$

In the limit $\Omega_{s} \rightarrow 0$, equation $\left(\mathrm{B} .22\right.$ reduces to $\left|a_{\alpha, \beta}\right|^{2} \delta_{\alpha, \beta}=1$. 


\section{Appendix C. Three Mode Mixing}

In this section we solve for the eigenvalues and eigenvectors of a three mode system coupled through the Coriolis force. We consider Mode 1 to be an f-mode, Mode 2 to be an arbitrary mode mixed with the f-mode, and Mode 3 to be an arbitrary mode that does not mix directly with Mode 1 but does mix with Mode 2. The eigensystem describing these three modes is

$$
\left[\begin{array}{ccc}
\left(\overline{\omega_{1}}-\omega\right) & C_{12} & 0 \\
C_{12}^{*} & \left(\overline{\omega_{2}}-\omega\right) & C_{23} \\
0 & C_{23}^{*} & \left(\overline{\omega_{3}}-\omega\right)
\end{array}\right]\left[\begin{array}{l}
b_{1} \\
b_{2} \\
b_{3}
\end{array}\right]=0,
$$

with $\bar{\omega}_{1}=\omega_{1}+C_{11}$ and likewise for modes 2 and 3 . This eigensystem is equivalent to

$$
\left[\begin{array}{ccc}
\Delta_{13}-\Delta & 2 C_{12} & 0 \\
2 C_{12}^{*} & \Delta_{2}-\Delta & 2 C_{23} \\
0 & 2 C_{23}^{*} & -\Delta_{13}-\Delta
\end{array}\right]\left[\begin{array}{l}
b_{1} \\
b_{2} \\
b_{3}
\end{array}\right]=0,
$$

where $\Delta_{13}=\bar{\omega}_{1}-\bar{\omega}_{3}, \Delta_{2}=2 \bar{\omega}_{2}-\bar{\omega}_{1}-\bar{\omega}_{3}$, and $\Delta=2 \omega-\bar{\omega}_{1}-\bar{\omega}_{3}$.

We now examine the specific case where modes 1 and 3 are degenerate, i.e., $\Delta_{13}=0$. The characteristic equation for this case is

$$
\Delta\left[\Delta^{2}-\Delta_{2} \Delta-4\left(\left|C_{12}\right|^{2}+\left|C_{23}\right|^{2}\right)\right]=0 .
$$

The eigenvalues are $\Delta_{0}=0$ and $\Delta_{ \pm}=\frac{1}{2}\left[\Delta_{2} \pm \sqrt{\Delta_{2}^{2}+16\left(\left|C_{12}\right|^{2}+\left|C_{23}\right|^{2}\right)}\right]$. These frequencies correspond to $\omega^{(0)}=\bar{\omega}_{1}=\bar{\omega}_{3}$ and

$$
\omega^{( \pm)}=\frac{2 \bar{\omega}_{2}+\bar{\omega}_{1}+\bar{\omega}_{3}}{4} \pm \frac{1}{4}\left[\Delta_{2}^{2}+16\left(\left|C_{12}\right|^{2}+\left|C_{23}\right|^{2}\right)\right]^{1 / 2} .
$$

The associated (unnormalized) eigenvectors are

$$
\mathbf{b}^{(0)}=\left[\begin{array}{c}
C_{23} \\
0 \\
-C_{12}^{*}
\end{array}\right]
$$

and

$$
\mathbf{b}^{( \pm)}=\left[\begin{array}{c}
2 C_{12} \\
\Delta_{ \pm} \\
2 C_{23}^{*}
\end{array}\right] .
$$

In all cases, the mode with frequency $\omega^{(0)}$ is a superposition of Modes 1 and 3, with the relative weights determined by the relative values of $\left|C_{12}\right|$ and $\left|C_{23}\right|$. If $\left|C_{12}\right| \ll\left|C_{23}\right|$, i.e., Mode 1 is essentially decoupled from the system, then this mode is a slightly perturbed version of Mode 1 . If $\left|C_{12}\right| \gg\left|C_{23}\right|$, i.e., Mode 3 is essentially decoupled from the system, then this mode is a slightly perturbed version of Mode 3 .

If the modes are minimally mixed, $\left[16\left(\left|C_{12}\right|+\left|C_{23}\right|\right) \ll\left|\Delta_{2}\right|\right]$, two modes have frequency $\omega \simeq \bar{\omega}_{1}=\bar{\omega}_{3}$ and one has $\omega \simeq \bar{\omega}_{2}$, as we would expect for non-mixed modes. However, the two modes with $\omega \simeq \bar{\omega}_{1}=\bar{\omega}_{3}$ may still be strongly mixed. In the nearly degenerate limit $\left[16\left(\left|C_{12}\right|+\left|C_{23}\right|\right) \gg\left|\Delta_{2}\right|\right]$, all three modes are mixed with one another, with the weights determined by the relative coupling coefficients.

It is important to note that if $\left|C_{12}\right|$ and $\left|C_{23}\right|$, have similar magnitudes, there will always exist at least two modes that are strong superpositions of Modes 1 and 3 , regardless of the value of $\Delta_{2}$. This entails that if a mode (in our example Mode 3 ) is nearly degenerate with the f-mode, it can mix strongly with it (through another mode, in our example Mode 2) even if it does not mix directly with the f-mode. The mode that serves as intermediary (Mode 2) need not be similar in frequency to Mode 1 and 3. In Section 4.2, we show that systems in which $\left|C_{12}\right|$ and $\left|C_{23}\right|$ have very different magnitudes can still exhibit strong mixing between Modes 1 and 3. In this case, the strong mixing does not occur when $\bar{\omega}_{1} \simeq \bar{\omega}_{3}$, although it does occur when there are two eigenfrequencies with $\omega \simeq \bar{\omega}_{1}$. We should therefore expect that modes very near in frequency to the f-mode will mix strongly with it, regardless of their original characteristics. 


\section{Appendix D. Effect of Modes on the Rings}

The characteristics of density waves launched at Lindblad resonances are characterized in Goldreich \& Tremaine (1979) and in Greenberg \& Brahic (1984). Here we summarize the relevant results and apply them to Saturn's ring system. The wave-like response of the disk near (but not exactly at) the Lindblad resonance is a traveling wave of the form

$$
\delta \Phi \simeq-\Psi \sqrt{2 \pi|z|} \exp \left[i x^{2} /(2 z)\right] .
$$

Here, $\delta \Phi$ is the wave-like gravitational perturbation produced in the rings (not to be confused with $\delta \Phi_{\alpha}$ or $\delta \Phi^{(\alpha)}$ associated with a given oscillation mode), $\Psi$ is the effective perturbing potential (see equation D.4), $x=\left(r-r_{L}\right) / r_{L}$ is the fractional distance away from the Lindblad resonance, and

$$
z=\frac{2 \pi G \Sigma}{r \mathcal{D}},
$$

is approximately the square of the radial wavelength. Here, $\Sigma$ is the unperturbed ring surface density, and $\mathcal{D} \simeq 3(m-1) \Omega^{2}$ near the Lindblad resonance. The value of $z$ is of order $z \sim M_{\text {rings }} / M \sim 10^{-9}$, where $M_{\text {rings }}$ is the mass of the C-ring. The wavelength is very short compared to the Lindblad radius, thus, the waves are very tightly wound. The wavelength decreases away from the Lindblad radius, although the waves typically damp out after $\sim 10$ wavelengths. This form of the response is accurate for $c_{s} /(\Omega r) \ll 1$, $G \Sigma /\left(\Omega^{2} r\right) \ll 1, c_{s}^{2} /(G \Sigma r) \ll 1$, where $c_{s}$ is the typical random velocity of ring particles. These are all excellent approximations for Saturn's rings.

A fairly accurate location of the resonant location of a mode is obtained by solving equation (22), including corrections due to the gravitational moments of Saturn. The solution is

$$
r_{L} \simeq r_{L 0}\left[1-\frac{1+m}{2(1-m)} J_{2}\left(\frac{R}{r_{0}}\right)^{2}+\frac{5(3+m)}{8(1-m)} J_{4}\left(\frac{R}{r_{0}}\right)^{4}\right]+\mathcal{O}\left(J_{6}, J_{2}^{2}\right),
$$

where $r_{L 0}$ is given by equation $\sqrt{23}$, and for Saturn $J_{2}=1.633 \times 10^{-2}$ and $J_{4}=-9.2 \times 10^{-4}$ (Guillot 2005). The effective forcing potential is

$$
\Psi=A\left[\frac{d}{d \ln r}+\frac{2 m \Omega}{m \Omega+\sigma_{\alpha}}\right] \delta \Phi_{\alpha}\left(r_{L}\right)
$$

where $A$ is the mode amplitude. Each component $l^{\prime}$ of the potential has the form $\delta \Phi_{\alpha, l^{\prime}} \propto r^{-\left(l^{\prime}+1\right)}$, resulting in $\Psi_{\alpha, l^{\prime}}=\left(2 m^{\prime}-l^{\prime}-1\right) \delta \Phi_{\alpha, l^{\prime}}$ (see also equation 25).

The associated density perturbation is

$$
\begin{aligned}
\delta \Sigma & \simeq \frac{i}{2 \pi G r^{1 / 2}} \frac{d}{d r}\left(r^{1 / 2} \delta \Phi\right) \\
& \simeq\left|\frac{3(1-m)}{4 \pi^{2}} \frac{\Omega^{2}|\Psi|^{2}}{G^{3} \Sigma r_{L}}\right|^{1 / 2} x \exp \left(i\left[x^{2} /(2 z)+m \phi+\sigma t\right]\right) .
\end{aligned}
$$

This equation applies in the inviscid limit and shows that the density perturbation becomes larger with increasing distance away from resonance. In reality, the waves damp out and (in Saturn's rings) the density perturbations typically decrease after one or two wavelengths away from resonance ${ }^{12}$ The associated perturbation in optical depth is $\delta \tau=\kappa_{m} \delta \Sigma$, where $\kappa_{m}$ is the local mass extinction coefficient (opacity) of the rings, and we have assumed that it is independent of $\Sigma$.

\footnotetext{
${ }^{12}$ Equation D.5 applies in the linear regime. Detectable waves in Saturn's rings often have density perturbations of order unity and are relatively non-linear, causing their optical depth variations to be cuspy. We ignore this issue in this work, although it may affect estimates of mode amplitude.
} 
We would like to use the observed variation in optical depth to estimate the amplitudes of the modes driving the waves. Since the wave amplitude is damped by viscous effects, we must judiciously choose a location $x$ at which to evaluate equation (D.5). This location must be far enough from the resonance such that equation (D.1) is applicable, but close enough so that the wave has not been significantly damped. Evaluating equation (D.5) at $x=\Delta r / r_{L}$, we have

$$
|\delta \tau| \approx\left|\frac{3(1-m)}{4 \pi^{2}} \frac{\kappa_{m}^{2} \Omega^{2}|\Psi|^{2}}{G^{3} \Sigma r_{L}}\right|^{1 / 2}\left|\frac{\Delta r}{r_{L}}\right|,
$$

Thus, the amplitude of the mode is

$$
|A| \approx\left|\frac{3(1-m)}{4 \pi^{2}} \frac{\kappa_{m}^{2} \Omega^{2}|\bar{\Psi}|^{2}}{G^{3} \Sigma r_{L}}\right|^{-1 / 2}\left|\frac{r_{L}}{\Delta r}\right||\delta \tau|,
$$

with

$$
\bar{\Psi}=\sum_{\beta} a_{\beta} W_{\beta} \delta \Phi_{\beta}(R)
$$

To estimate mode amplitudes from HN13, we typically evaluate $\delta \tau$ near its maximum, about one wavelength away from the resonant radius, at $\Delta r \sim 5 \mathrm{~km}$. This procedure assumes that damping has had a negligible effect on wave amplitude within the first wavelength.

Finally, to calculate optical depth fluctuations produced by modes in our Saturn models, we first calculate the amplitude of a mode required to produce the largest observed $m=-3$ wave in Saturn's rings. We use the observed values of $\kappa_{m}$ and $\sigma_{m}$ from HN13, and we assume the wave is generated by an unmixed $l=3$, $m=-3$ f-mode with $\delta \Phi_{\alpha}(R) \sim 1$, determined from our mode calculations. This procedure typically results in a dimensionless amplitude $\left|A_{3}\right| \simeq 10^{-9}$. We then assume energy equipartition amongst the oscillation modes, such that the amplitudes of modes in our model are determined by $\omega_{\alpha}^{2}\left|A_{\alpha}\right|^{2}=\omega_{3}^{2}\left|A_{3}\right|^{2}$. The optical depth variation is then calculated according to equation (D.6), and is evaluated at $\Delta r \sim 5 \mathrm{~km}$. 